\title{
Multicluster Interleaving on Paths and Cycles
}

\author{
Anxiao (Andrew) Jiang, Member, IEEE, and Jehoshua Bruck, Fellow, IEEE
}

\begin{abstract}
Interleaving codewords is an important method not only for combatting burst errors, but also for distributed data retrieval. This paper introduces the concept of multicluster interleaving (MCI), a generalization of traditional interleaving problems. MCI problems for paths and cycles are studied. The following problem is solved: how to interleave integers on a path or cycle such that any $m(m \geq 2)$ nonoverlapping clusters of order 2 in the path or cycle have at least three distinct integers. We then present a scheme using a "hierarchical-chain structure" to solve the following more general problem for paths: how to interleave integers on a path such that any $m(m \geq 2)$ nonoverlapping clusters of order $L(L \geq 2)$ in the path have at least $L+1$ distinct integers. It is shown that the scheme solves the second interleaving problem for paths that are asymptotically as long as the longest path on which an MCI exists, and clearly, for shorter paths as well.
\end{abstract}

Index Terms-Burst error, cluster, cycle, file placement, interleaving, multicluster interleaving (MCI), path.

\section{INTRODUCTION}

I NTERLEAVING codewords is an important method for both combatting burst errors and distributed data retrieval. Every interleaving scheme can be interpreted as labeling a graph's vertices with integers, and traditional interleaving problems all focus on local properties of the labeling. Specifically, if we define a cluster to be a connected subgraph of certain characteristics (such as size, shape, etc., depending on the specific definition of the interleaving problem), then traditional interleaving problems require that in every single cluster, the number of different integers exceeds a threshold, or every integer appears less than a certain number of times, etc.

Applications of interleaving in burst-error correction are well known. The most familiar example is the interleaving of codewords on a path, which has the form " $1,2,3, \ldots n$, $1,2,3, \ldots n, \ldots$," for combatting one-dimensional burst errors of length up to $n$. This one-dimensional interleaving is generalized to higher dimensions in [3]-[5] and [7], where integers are used to label the vertices of a two-dimensional or higher dimensional array in such a way that in every connected subgraph of order $t$ of the array, each integer appears at most $r$ times. ( $t$ and $r$ here are parameters. The order of a graph is defined

Manuscript received August 17, 2003; revised July 29, 2004. This work was supported in part by the Lee Center for Advanced Networking at the California Institute of Technology, and by the National Science Foundation under Grant CCR-TC-0209042. The material in this paper was presented in part at the 7th International Symposium on Communication Theory and Applications, Ambleside, Lake District, UK, July 2003.

The authors are with the Department of Electrical Engineering, California Institute of Technology, MC 136-93, Pasadena, CA 91125 USA (e-mail: jax@paradise.caltech.edu; bruck@paradise.caltech.edu).

Communicated by K. A. S. Abdel-Ghaffar, Associate Editor for Coding Theory.

Digital Object Identifier 10.1109/TIT.2004.840893 as the number of vertices in that graph.) More work on such a generalized interleaving scheme includes [10], [12], [15]-[17], where the underlying graphs on which integers are interleaved include tori, arrays, and circulant graphs. In [1]-[3], codewords are interleaved on arrays to correct burst errors of rectangular shapes, circular shapes, or arbitrary connected shapes.

Applications of interleaving in distributed data retrieval, although maybe less well known, are just as broad. Data streaming and broadcast schemes using erasure-correcting codes have received extensive interest in both academia and industry, where interleaved components of a codeword are transmitted in sequence, and every client can listen to this data flow for a while until enough codeword components are received for recovering the information [6], [11]. (An example is shown in Fig. 1(a), where a codeword of seven components is broadcast repeatedly. We assume that the codeword can tolerate two erasures. Therefore, every client only needs to receive five different components. In this example, the codeword components can be understood as interleaved on a path or a cycle.) Interleaving is also studied in the scenario of file retrieval in networks, where a file is encoded into a codeword, and components of the codeword are interleavingly placed on a network, such that every node in the network can retrieve enough distinct codeword components from its proximity for recovering the file [9], [13]. (An example is shown in Fig. 1(b), where the codeword again has length 7 and can tolerate two erasures. We assume that all edges have length 1 . Then every network node can retrieve five distinct codeword components from its proximity of radius 2 for recovering the file.)

This paper introduces the concept of multicluster interleaving (MCI). In general, an MCI problem is concerned with labeling the vertices of a given graph in such a way that for any $m$ clusters, the integers in them are sufficiently diversified (by certain criteria). Traditional interleaving problems correspond to the case $m=1$. So MCI is a natural extension of the traditional concept of interleaving.

We focus on MCI on paths and cycles. In this paper, we study the following problem.

Definition 1: Let $G=(V, E)$ be a path (or cycle) of $n$ vertices. Let $N, K, m$, and $L$ be positive integers such that $N \geq K>L$ and $m \geq 2$. A cluster is defined to be a connected subgraph of order $L$ of the path (or cycle). Assign one integer in the set $\{1,2, \ldots, N\}$ to each vertex. Such an assignment is called a multicluster interleaving (MCI) if and only if every $m$ nonoverlapping clusters have no less than $K$ distinct integers.

The above MCI problem is fully characterized by the five parameters- $n, N, K, m, L-$ and the graph $G=(V, E)$. We note that throughout this paper, the parameters $n, N, K, m, L$, 
(a) Broadcast

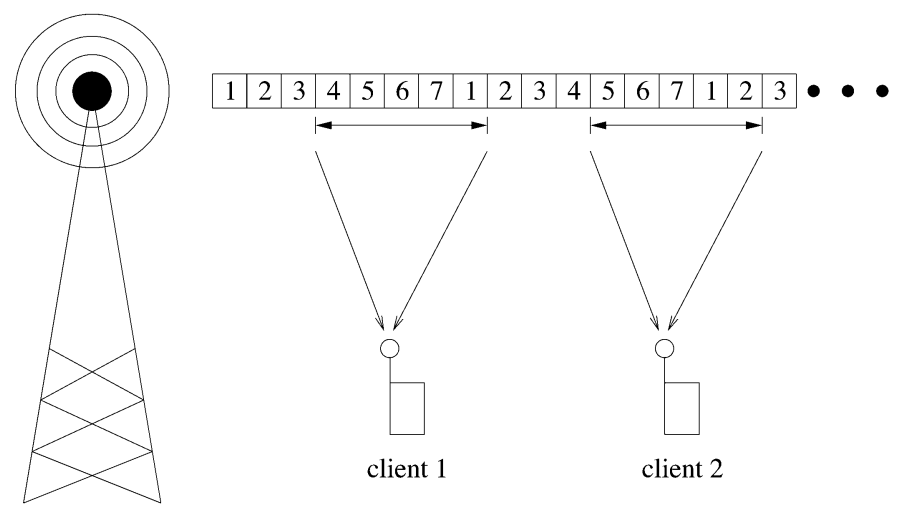

(b) File storage in a network

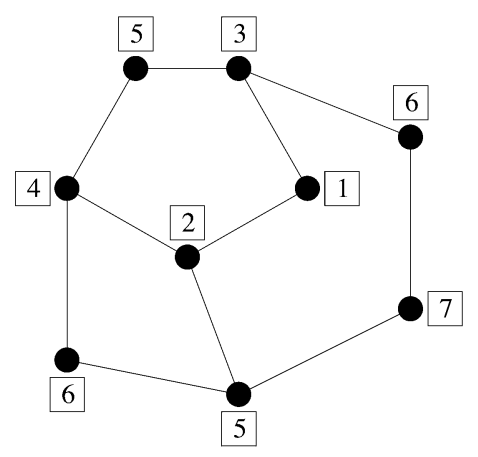

Fig. 1. Examples of interleaving for data retrieval.

and the graph $G=(V, E)$ will always have the meanings as defined in Definition 1.

The following is an example of the MCI problem.

Example 1: A cycle of $n=21$ vertices is shown in Fig. 2. The parameters are $N=9, K=5, m=2$, and $L=3$. An interleaving is shown in the figure, where the integer on every vertex is the integer assigned to it. It can be verified that any 2 nonoverlapping clusters of order 3 have at least 5 distinct integers. For example, the two clusters in dashed circles have integers " $9,1,2$ " and " $7,1,6$," respectively, so they together have 5 distinct integers: $1,2,6,7,9$. So the interleaving is an MCI on the cycle.

If we remove an edge in the cycle, then it will become a path. Clearly, if all other parameters remain the same, the interleaving shown in Fig. 2 will be an MCI on the path.

MCI has applications in distributed data storage in networks and data retrieval by clients that are capable of accessing multiple parts of the network. The MCI problem defined in Definition 1 has the following interpretation. The $N$ integers used to label the vertices in the path/cycle represent the $N$ components in a codeword. $K$ is the minimum number of components needed for decoding the codeword. (In other words, the codeword can correct $N-K$ erasures.) An interleaving of the integers represents the placement of the codeword components on the path/cycle. For each client that wants to retrieve data from the path/cycle, we assume it can access $m$ nonoverlapping clus-

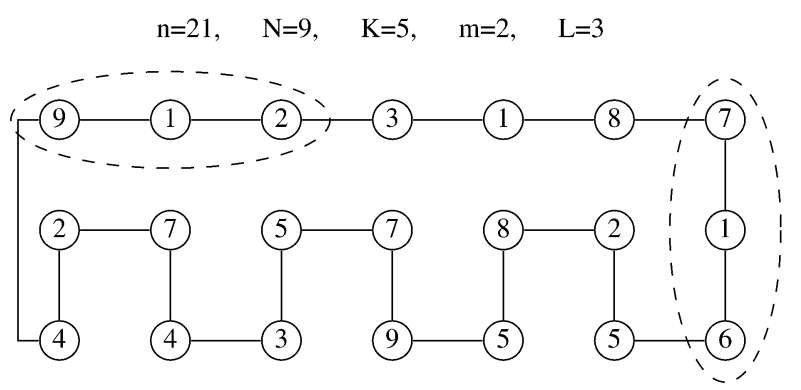

Fig. 2. An example of MCI.

ters; and we assume different clients can access different sets of clusters. (By imposing the restriction that the $m$ clusters a client can access must be nonoverlapping, we ensure that each client can access no less than $m L$ vertices.) Then when the interleaving is an MCI, every client can retrieve enough data for decoding the codeword.

MCI on paths and cycles appears to have natural applications in data streaming and broadcast [8]. Imagine that the components of a codeword interleaved the same way are transmitted asynchronously in several channels. Then, a client can simultaneously listen to multiple channels in order to get data faster, which is equivalent to retrieving data from multiple clusters. Another possible application is data storage on disks [14], where we assume multiple heads can read different parts of a disk in parallel to accelerate input/output (I/O) speed.

The MCI problem for paths and cycles can be divided into smaller problems based on the values of the parameters. The key results of this paper are as follows.

- The family of problems with the constraints that $L=2$ and $K=3$ are solved for both paths and cycles. We show that when $L=2$ and $K=3$, an MCI exists on a path if and only if the number of vertices in the path is no greater than $(N-1)[(m-1) N-1]+2$, and an MCI exists on a cycle if and only if the number of vertices in the cycle is no greater than $(N-1)[(m-1) N-1]$. Structural properties of MCIs in this case are analyzed, and algorithms are presented which can output MCIs on paths and cycles as long as the MCIs exist.

- The family of problems with the constraint that $K=L+1$ are studied for paths. A scheme using a "hierarchicalchain" structure is presented for constructing MCIs. It is shown that the scheme solves the MCI problem for paths that are asymptotically as long as the longest path on which MCIs exist, and clearly, for shorter paths as well.

The rest of the paper is organized as follows. In Section II, we derive an upper bound for the orders of paths and cycles on which MCIs exist. We then prove a tighter upper bound for paths for the case of $L=2$ and $K=3$. In Section III, we present an optimal construction for MCI on paths for the case of $L=2$ and $K=3$, which meets the upper bound presented in Section II. In Section IV, we study the MCI problem for paths when $K=L+1$. In Section $\mathrm{V}$, we extend our results from paths to cycles. In Section VI, we conclude this paper. 


\section{UPPER BOUNDS}

While traditional one-dimensional interleaving exists on infinitely long paths, that is no longer true for MCI. If $K=m L$, then to get an MCI, every integer can be assigned to only one vertex of the path/cycle, which means that MCI exists only for paths/cycles of order $N$ or less. When $K$ has smaller values, MCI exists for longer paths/cycles. The following proposition presents an upper bound for the orders of paths/cycles.

Proposition 1: If an MCI exists on a path (or cycle) of $n$ vertices, then

$$
n \leq(m-1) L\left(\begin{array}{c}
N \\
L
\end{array}\right)+(L-1) .
$$

Proof of Proposition 1: Let $G=(V, E)$ be a path (or cycle) of $n$ vertices with an MCI on it. $G$ contains at most $\left\lfloor\frac{n}{L}\right\rfloor$ nonoverlapping clusters. Let $S \subseteq\{1,2, \ldots, N\}$ be an arbitrary set of $L$ distinct integers. Then since the interleaving on $G$ is an MCI, among those $\left\lfloor\frac{n}{L}\right\rfloor$ nonoverlapping clusters, at most $m-1$ of them are assigned only integers in $S . S$ can be one of $\left(\begin{array}{l}N \\ L\end{array}\right)$ possible sets. So

$$
\left\lfloor\frac{n}{L}\right\rfloor \leq(m-1)\left(\begin{array}{c}
N \\
L
\end{array}\right)
$$

and hence,

$$
n \leq(m-1) L\left(\begin{array}{c}
N \\
L
\end{array}\right)+(L-1) .
$$

Note that for the same set of parameters $N, K, m$, and $L$, if MCI exists on a path of $n=n_{0}$ vertices, then it also exists on any path of $n<n_{0}$ vertices. That is because given an MCI on a path, by removing vertices from the ends of the path, we can get MCIs on shorter paths. However, such an argument does not necessarily hold for cycles.

The upper bound of Proposition 1 is in fact loose. For example, when $N=K=3$ and $m=L=2$, a simple exhaustive search will show that an MCI exists on a path (or a cycle) if and only if the path (respectively, cycle) is of order 6(respectively, 4) or less. However, Proposition 1 gives an upper bound which is

$$
n \leq(m-1) L\left(\begin{array}{c}
N \\
L
\end{array}\right)+(L-1)=7 .
$$

In the remainder of this section, we shall prove a tighter upper bound for paths for the case of $L=2$ and $K=3$, stated as the following theorem. Later study will show that this bound is exact.

Theorem 1: When $L=2$ and $K=3$, if there exists an MCI on a path of $n$ vertices, then $n \leq(N-1)[(m-1) N-1]+2$.

Theorem 1 will be established by proving the following three lemmas. Before starting the formal analysis, we first define some notations that will be used throughout this paper. Let $G=(V, E)$ be a path. We denote the $n$ vertices in the path $G$ by $v_{1}, v_{2}, \ldots, v_{n}$. For $2 \leq i \leq n-1$, the two vertices adjacent to $v_{i}$ are $v_{i-1}$ and $v_{i+1}$. A connected subgraph of $G$ induced by vertices $v_{i}, v_{i+1}, \ldots, v_{j}(j \geq i)$ is denoted by $\left(v_{i}, v_{i+1}, \ldots, v_{j}\right)$. If a set of integers are interleaved on $G$, then $c\left(v_{i}\right)$ denotes the integer assigned to vertex $v_{i}$.
The following lemma reveals a structural property of MCI.

Lemma 1: Let the values of $N, K, m$, and $L$ be fixed, where $N \geq 4, K=3, m \geq 2$, and $L=2$. Let $n_{\max }$ denote the maximum value of $n$ such that an MCI exists on a path of $n$ vertices. Then in any MCI on a path of $n_{\max }$ vertices, no two adjacent vertices are assigned the same integer.

Proof of Lemma 1: Let $G=(V, E)$ be a path of $n_{\max }$ vertices with an MCI on it, and assume two adjacent vertices of $G$ are assigned the same integer. We will prove that an MCI exists on a path of more than $n_{\max }$ vertices, which is a contradiction.

Without loss of generality (WLOG), one of the following four cases must be true (because we can always get one of the four cases by permuting the names of the integers and by reversing the indices of the vertices):

Case 1: There exist four consecutive vertices in $G-$ $v_{i}, v_{i+1}, v_{i+2}, v_{i+3}$-such that $c\left(v_{i}\right)=1$, $c\left(v_{i+1}\right)=c\left(v_{i+2}\right)=2, c\left(v_{i+3}\right)=1$ or 3 .

Case 2: There exist $x+2 \geq 5$ consecutive vertices in $G$ $v_{i}, v_{i+1}, \ldots, v_{i+x}, v_{i+x+1}$-such that $c\left(v_{i}\right)=1$, $c\left(v_{i+1}\right)=c\left(v_{i+2}\right)=\cdots=c\left(v_{i+x}\right)=2$, $c\left(v_{i+x+1}\right)=1$ or 3 .

Case 3: $\quad c\left(v_{1}\right)=c\left(v_{2}\right)=1, c\left(v_{3}\right)=2$.

Case 4: $\quad c\left(v_{1}\right)=c\left(v_{2}\right)=\cdots=c\left(v_{x}\right)=1$ and $c\left(v_{x+1}\right)=$ 2 , where $x \geq 3$.

We analyze the four cases one by one.

Case 1: In this case, we insert a vertex $v^{\prime}$ between $v_{i+1}$ and $v_{i+2}$, and get a new path of $n_{\max }+1$ vertices. Call this new path $H$, and assign the integer " 4 " to $v^{\prime}$. Consider any $m$ nonoverlapping clusters in $H$. If none of those $m$ clusters contains $v^{\prime}$, then clearly they are also $m$ nonoverlapping clusters in the path $G$, and, therefore, have been assigned at least $K=$ 3 distinct integers. If the $m$ clusters contain all the three vertices $v_{i+1}, v^{\prime}$, and $v_{i+2}$, then they also contain either $v_{i}$ or $v_{i+3}$-therefore, they have been assigned at least $K=3$ distinct integers: " $1,2,4$ " or “" $2,3,4$." WLOG, the only remaining possibility is that one of the $m$ clusters contains $v_{i+1}$ and $v^{\prime}$ while none of them contains $v_{i+2}$. Note that among the $m$ clusters, the $m-1$ of them which do not contain $v^{\prime}$ are also $m-1$ clusters in the path $G$, and they together with $\left(v_{i+1}, v_{i+2}\right)$ are $m$ nonoverlapping clusters in $G$ and, therefore, are assigned at least $K=3$ distinct integers. Since $c\left(v_{i+1}\right)=c\left(v_{i+2}\right)$, the original $m$ clusters including $\left(v_{i+1}, v^{\prime}\right)$ must also have been assigned at least $K=3$ distinct integers. So $H$ has $n_{\max }+1$ vertices and has an MCI on it, which is a contradiction.

Case 2: In this case, we insert a vertex $v^{\prime}$ between $v_{i+1}$ and $v_{i+2}$, and insert a vertex $v^{\prime \prime}$ between $v_{i+x-1}$ and $v_{i+x}$, and get a new path of $n_{\max }+2$ vertices. Call this new path $H$, assign the integer " 4 " to $v^{\prime}$, and assign the integer " 3 " to $v^{\prime \prime}$. Consider any $m$ nonoverlapping clusters in $H$. If the $m$ clusters contain neither $v^{\prime}$ nor $v^{\prime \prime}$, then clearly they are also $m$ nonoverlapping clusters in the path $G$, and, 
therefore, are assigned at least $K=3$ distinct integers. If the $m$ clusters contain both $v^{\prime}$ and $v^{\prime \prime}$, then they also contain at least one vertex in the set $\left\{v_{i+1}, v_{i+2}, \ldots, v_{i+x-1}, v_{i+x}\right\}$, and therefore are assigned at least these three integers: " 2, , " 3 ," and "4." WLOG, the only remaining possibility is that the $m$ clusters contain $v^{\prime}$ but not $v^{\prime \prime}$. (Note that the cluster containing $v^{\prime}$ is assigned integers " 2 " and "4.") When that possibility is true, if the $m$ clusters contain $v_{i+x+1}$, then they are assigned at least three distinct integers-" $1,2,4$ " or " $2,3,4$." If the $m$ clusters do not contain $v_{i+x+1}$, then they do not contain $v_{i+x}$ either-then we divide the $m$ clusters into two groups $A$ and $B$, where $A$ is the set of clusters none of which contains any vertex in $\left\{v^{\prime}, v_{i+2}, v_{i+3}, \ldots, v_{i+x-1}\right\}$, and $B$ is the complement set of $A$. Say there are $y$ clusters in $B$. Then, if the cluster containing $v^{\prime}$ also contains $v_{i+1}$ (respectively, $v_{i+2}$ ), there exists a set $C$ of $y$ clusters in the path $G$ that only contain vertices in $\left\{v_{i+1}, v_{i+2}, \ldots, v_{i+x-1}, v_{i+x}\right\}$ (respectively, $\left.\left\{v_{i+2}, v_{i+3}, \ldots, v_{i+x-1}, v_{i+x}\right\}\right)$, such that the $m$ clusters in $A \cup C$ are nonoverlapping in $G$. Those $m$ clusters in $A \cup C$ are assigned at least $K=3$ distinct integers since the interleaving on $G$ is an $\mathrm{MCI}$; and they are assigned no more distinct integers than the original $m$ clusters in $A \cup B$ are, because $c\left(v_{i+1}\right)=c\left(v_{i+2}\right)=\cdots=c\left(v_{i+x}\right)$ and either $v_{i+1}$ or $v_{i+2}$ is in the same cluster containing $v^{\prime}$. So the $m$ clusters in $A \cup B$ are assigned at least $K=3$ distinct integers. So $H$ has $n_{\max }+2$ vertices and has an MCI on it, which is again a contradiction.

Case 3: In this case, we insert a vertex $v^{\prime}$ between $v_{1}$ and $v_{2}$, and assign the integer " 3 " to $v^{\prime}$. The rest of the analysis is very similar to that for Case 1.

Case 4: In this case, we insert a vertex $v^{\prime}$ between $v_{1}$ and $v_{2}$, and insert a vertex $v^{\prime \prime}$ between $v_{x-1}$ and $v_{x}$, assign the integer " 3 " to $v^{\prime}$, and assign the integer " 2 " to $v^{\prime \prime}$. The rest of the analysis is very similar to that for Case 2.

So a contradiction exists in all the four cases. Therefore, this lemma is proved.

The next two lemmas derive upper bounds for paths, respectively, for the case " $N \geq 4$ " and the case " $N=3$."

Lemma 2: Let the values of $N, K, m$, and $L$ be fixed, where $N \geq 4, K=3, m \geq 2$, and $L=2$. Let $n_{\max }$ denote the maximum value of $n$ such that an MCI exists on a path of $n$ vertices. Then $n_{\max } \leq(N-1)[(m-1) N-1]+2$.

Proof of Lemma 2: Let $G=(V, E)$ be a path of $n_{\max }$ vertices. Assume there is an MCI on $G$. By Lemma 1, no two adjacent vertices in $G$ are assigned the same integer. We color the vertices in $G$ with three colors-red, yellow, and green-through the following three steps.

Step 1) for $2 \leq i \leq n_{\max }-1$, if $c\left(v_{i-1}\right)=c\left(v_{i+1}\right)$, then color $\bar{v}_{i}$ with the red color;

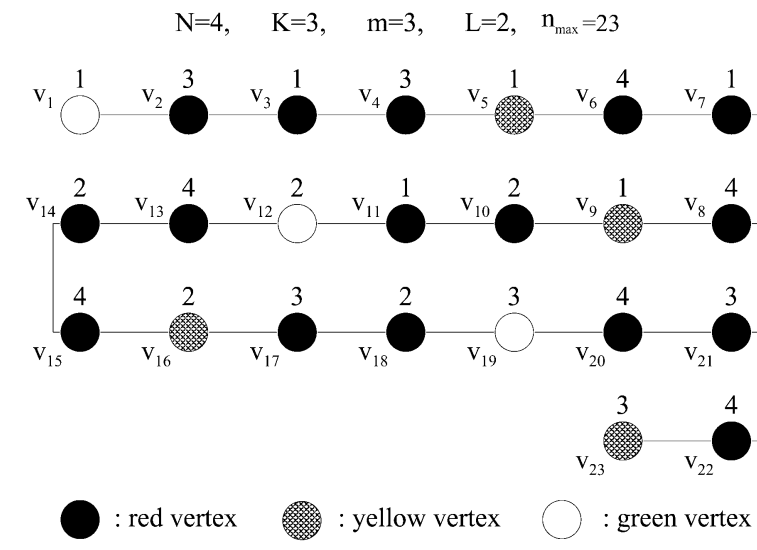

Fig. 3. In this example, $N=4, K=3, m=3, L=2$. An oracle tells us that $n_{\max }=23$. Let $G=(V, E)$ be the path shown in the figure, which has 23 vertices and an MCI on it. Then the vertices of $G$ will be colored to be red, yellow, and green as shown.

Step 2) for $2 \leq i \leq n_{\max }$, color $v_{i}$ with the yellow color if $v_{i}$ is not colored red and there exists $j$ such that these four conditions are satisfied: 1) $1 \leq j<i$,2) $v_{j}$ is not colored red, 3) $\left.c\left(v_{j}\right)=c\left(v_{i}\right), 4\right)$ the vertices between $v_{j}$ and $v_{i}$-that is, $v_{j+1}, v_{j+2}, \ldots, v_{i-1}$-are all colored red;

Step 3) for $1 \leq i \leq n_{\max }$, if $v_{i}$ is neither colored red nor colored yellow, then color $v_{i}$ with the green color.

Clearly, each vertex of $G$ is assigned exactly one of the three colors. (See Fig. 3 for an example.)

If we arbitrarily pick two different integers-say " $i$ " and " $j "$-from the set $\{1,2, \ldots, N\}$, then we get a pair $[i, j]$ (or $[j, i]$, equivalently). There are totally $\left(\begin{array}{c}N \\ 2\end{array}\right)$ such unordered pairs. We partition those $\left(\begin{array}{c}N \\ 2\end{array}\right)$ pairs into four groups " $A$, , " $B$," " $C$," and " $D$ " in the following way.

1) A pair $[i, j]$ belongs to group $A$ if and only if the following two conditions are satisfied: i) at least one green vertex is assigned the integer " $"$ " and at least one green vertex is assigned the integer " $j$," ii) for any two green vertices that are assigned integers " $i$ " and " $j$," respectively, there is at least one green vertex between them.

2) A pair $[i, j]$ belongs to group $B$ if and only if the following two conditions are satisfied: i) at least one green vertex is assigned the integer " $~ "$ " and at least one green vertex is assigned the integer " $j$," ii) there exist two green vertices that are assigned integers " $i$ " and " $j$," respectively, such that there is no green vertex between them.

3) A pair $[i, j]$ belongs to group $C$ if and only if one of the following two conditions is satisfied: i) at least one green vertex is assigned the integer " $i$ " and no green vertex is assigned the integer " $j$," ii) at least one green vertex is assigned the integer " $j$ " and no green vertex is assigned the integer " $i$."

4) A pair $[i, j]$ belongs to group $D$ if and only if no green vertex is assigned the integer " $i$ " or " $j$."

(See Fig. 4 for an example.) 
Group A: [1,3]

Group B: $[1,2],[2,3]$

Group C: $[1,4],[2,4],[3,4]$

Group D: empty.

Fig. 4. Let us continue the example in Fig. 3. Then groups $A, B, C, D$ are as shown here.

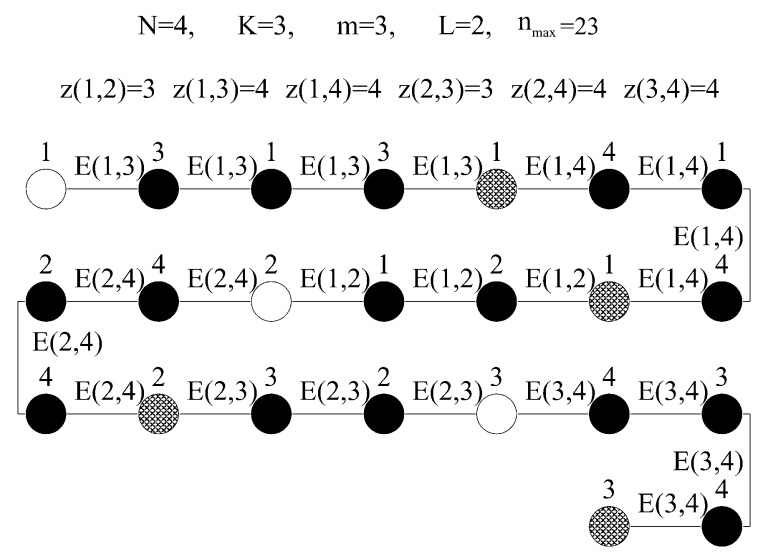

Fig. 5. Let us continue the example in Fig. 3. Then the set $E(i, j)$ that an edge belongs to is labeled beside that edge. The value of each $z(i, j)$ is shown in the figure.

For any $1 \leq i \neq j \leq N$, let $E(i, j) \subseteq E$ denote the following subset of edges of $G$ : an edge of $G$ is in $E(i, j)$ if and only if one endpoint of the edge is assigned the integer " $i$ " and the other endpoint of the edge is assigned the integer " $j$." Let $z(i, j)$ denote the number of edges in $E(i, j)$. (See Fig. 5 for an example.) In the following, we derive upper bounds for $z(i, j)$.

For any pair $[i, j]$ in group $A$ or group $C, z(i, j) \leq 2 m-2$. This is because otherwise there would exist $m$ nonoverlapping clusters in $G$ each of which is assigned only integers " $i$ " and " $j$," which would contradict the assumption that the interleaving on $G$ is an MCI. (See Fig. 6 for an example.)

Now consider a pair $[i, j]$ in group $B . z(i, j) \leq 2 m-2$ for the same reason as in the previous case. In the following, we will prove that $z(i, j) \leq 2 m-3$ by using a contradiction. Assume $z(i, j)=2 m-2$. Then in order to avoid the existence of $m$ nonoverlapping clusters in $G$ that are assigned only integers " $i$ " and " $j$," the $z(i, j)=2 m-2$ edges in $E(i, j)$ must be consecutive in the path $G$, which means, WLOG, that there are $2 m-1$ consecutive vertices $v_{y+1}, v_{y+2}, \ldots, v_{y+2 m-1}(y \geq 0)$ whose assigned integers are in the form of

$$
\left[c\left(v_{y+1}\right), c\left(v_{y+2}\right), \ldots, c\left(v_{y+2 m-1}\right)\right]=[i, j, i, j, \ldots, i, j, i]
$$

(See Fig. 7 for an example.)

According to the definition of "group $B$," there exist a green vertex $v_{k_{1}}$ and a green vertex $v_{k_{2}}$, such that $v_{k_{1}}$ is assigned the integer " $i, " v_{k_{2}}$ is assigned the integer " $j$," and there is no green vertex between them. Therefore, every vertex between $v_{k_{1}}$ and $v_{k_{2}}$ is either red or yellow. There are two possible cases.

Case 1: $k_{1}<k_{2}$. Then the path $G$ is interleaved as in Fig. 8(a). We use $v_{p_{1}}, v_{p_{2}}, \ldots, v_{p_{t}}$ to denote all the yellow vertices between $v_{k_{1}}$ and $v_{k_{2}}$. (The other vertices between $v_{k_{1}}$ and $v_{k_{2}}$ are all red.)
By the definition of "yellow vertices," we can see that

$c\left(v_{p_{t}}\right)=c\left(v_{p_{t-1}}\right)=\cdots=c\left(v_{p_{1}}\right)=c\left(v_{k_{1}}\right)=i$.

Since the vertices between $v_{p_{t}}$ and $v_{k_{2}}$ are all red, and the two vertices adjacent to any red vertex must be assigned the same integer, we can see that $c\left(v_{k_{2}-1}\right)=c\left(v_{p_{t}}\right)=i$. Since there is an edge between $v_{k_{2}-1}$ (which is assigned the integer " $i$ ") and $v_{k_{2}}$ (which is assigned the integer " $j$ "), $v_{k_{2}}$ must be in the set $\left\{v_{y+1}, v_{y+2}, \ldots, v_{y+2 m-1}\right\}$. However, it is simple to see that every vertex in the set $\left\{v_{y+1}, v_{y+2}, \ldots, v_{y+2 m-1}\right\}$ that is assigned the integer " $j$ " must be red —so $v_{k_{2}}$ should be red instead of green - therefore, a contradiction exists.

Case 2: $k_{2}<k_{1}$. Then the path $G$ is interleaved as in Fig. 8(b). We use $v_{p_{1}}, v_{p_{2}}, \ldots, v_{p_{t}}$ to denote all the yellow vertices between $v_{k_{2}}$ and $v_{k_{1}}$. (The other vertices between $v_{k_{2}}$ and $v_{k_{1}}$ are all red.)

We can see that $c\left(v_{k_{1}-1}\right)=j$. Since there is an edge between $v_{k_{1}-1}$ (which is assigned the integer " $j$ ") and $v_{k_{1}}$ (which is assigned the integer " $i$ "), both $v_{k_{1}-1}$ and $v_{k_{1}}$ are in the set $\left\{v_{y+2}, v_{y+3}, \ldots, v_{y+2 m-1}\right\}$. Since every vertex in the set $\left\{v_{y+1}, v_{y+2}, \ldots, v_{y+2 m-1}\right\}$ that is assigned the integer " $j$ " must be red, and since the color of $v_{k_{1}}$ is green, it is simple to see that all the vertices in the set $\left\{v_{y+1}, v_{y+2}, \ldots, v_{k_{1}-1}\right\}$ that are assigned the integer " $i$ " must be red (because otherwise $v_{k_{1}}$ would have to be yellow). Then since the color of $v_{y+1}$ is red, the vertex $v_{y}$ exists and it must have been assigned the integer " $c\left(v_{y+2}\right)=j$ "- and that contradicts the statement that all the edges in $E(i, j)$ are in the subgraph $\left(v_{y+1}, v_{y+2}, \ldots, v_{y+2 m-1}\right)$.

Therefore, a contradiction always exists when $z(i, j)=$ $2 m-2$. So for any pair $[i, j]$ in group $B, z(i, j) \leq 2 m-3$.

Now consider a pair $[i, j]$ in group $D$. By the definition of "group $D$," no green vertex is assigned the integer " $i$ " or " $j$." Let $\left\{v_{k_{1}}, v_{k_{2}}, \ldots, v_{k_{t}}\right\}$ denote the set of vertices that are assigned the integer " $i$," where $k_{1}<k_{2}<\cdots<k_{t}$. If $\left\{v_{k_{1}}, v_{k_{2}}, \ldots, v_{k_{t}}\right\} \neq \emptyset$, by the way vertices are colored, it is simple to see that $v_{k_{1}}$ cannot be yellow-so $v_{k_{1}}$ must be red. Then similarly, $v_{k_{2}}, v_{k_{3}}, \ldots, v_{k_{t}}$ must be $r e d$, too. Therefore, all the vertices that are assigned the integer " $i$ " are of the color $r e d$. Similarly, all the vertices that are assigned the integer " $j$ " are of the color red. Assume there is an edge whose two endpoints are assigned the integer " $i$ " and the integer " $j$," respectively. Then since the two vertices adjacent to any red vertex must be assigned the same integer, there exists an infinitely long subgraph of the path $G$ to which the assigned integers are in the form of "...i,j,i,j,i,j ..." which is certainly impossible. Therefore, a contradiction exists. So for any pair $[i, j]$ in group $D, z(i, j)=0$.

Let $x$ denote the number of distinct integers assigned to green vertices, and let $X$ denote the set of those $x$ distinct integers. It is simple to see that exactly $\left(\begin{array}{l}x \\ 2\end{array}\right)$ pairs $[i, j]$ are in group $A$ or group $B$, where $i \in X$ and $j \in X$-and among them at least $x-1$ pairs are in group $B$. It is also simple to see that exactly $x(N-x)$ pairs are in group $C$ and exactly $\left(\begin{array}{c}N-x \\ 2\end{array}\right)$ pairs are in 


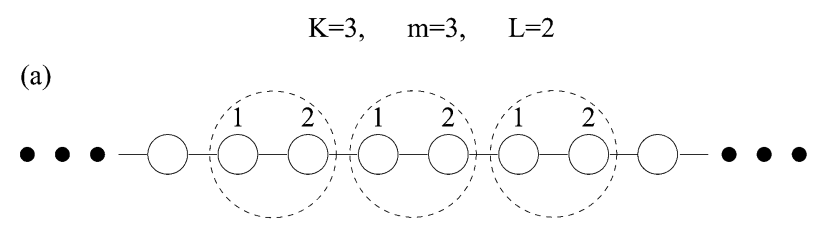

(b)

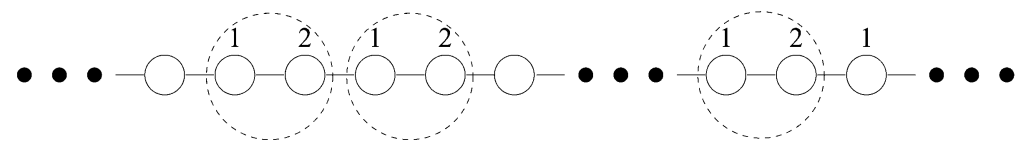

Fig. 6. In this example, $K=3, m=3, L=2$. Two paths are shown, respectively, in (a) and (b), each of which has more than $2 m-2=4$ edges in the set $E(1,2)$. Then both of them contain $m=3$ nonoverlapping clusters (as shown in dashed circles) that are assigned only two distinct integers, which proves that the interleaving on them cannot be MCI.

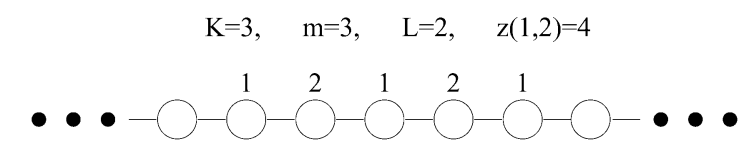

Fig. 7. In this example, $K=3, m=3, L=2, z(1,2)=2 m-2=4$. Then for a path with an MCI on it, the four edges whose endpoints are labeled by " 1 " and " 2 " have to be consecutive, as shown in the figure.

group $D$. By using the upper bounds we have derived for $z(i, j)$, we see that the number of edges in $G$ is at most s

$$
\begin{aligned}
{\left[\left(\begin{array}{l}
x \\
2
\end{array}\right)-(x-1)\right] \cdot(2 m-2)+(x-1) \cdot(2 m-3) } \\
+x(N-x) \cdot(2 m-2)+\left(\begin{array}{c}
N-x \\
2
\end{array}\right) \cdot 0 \\
=(1-m) x^{2}+(2 m N-2 N-m) x+1
\end{aligned}
$$

whose maximum value (at integer solutions) is achieved when $x=N-1$-and that maximum value is $(N-1)[(m-1) N-$ $1]+1$. So $n_{\max }$, the number of vertices in $G$, is at most $(N-$ 1) $[(m-1) N-1]+2$.

Lemma 3: Let the values of $N, K, m$, and $L$ be fixed, where $N=3, K=3, m \geq 2$, and $L=2$. Let $n_{\max }$ denote the maximum value of $n$ such that an MCI exists on a path of $n$ vertices. Then $n_{\max } \leq(N-1)[(m-1) N-1]+2$.

Proof of Lemma 3: Let $G=(V, E)$ be a path of $n$ vertices that has an MCI on it. We need to show that

$$
n \leq(N-1)[(m-1) N-1]+2 .
$$

If no two adjacent vertices of $G$ are assigned the same integer, then with the same argument as in the proof of Lemma 2, it can be shown that $n \leq(N-1)[(m-1) N-1]+2$.

Now assume two adjacent vertices of $G$ are assigned the same integer. Clearly, we can find $t$ nonoverlapping clusters in $G$, such that $n \leq 2 t+2$ and at least one of the $t$ clusters contains two vertices that are assigned the same integer. Among those $t$ nonoverlapping clusters, let $x, y, z, a, b$, and $c$, respectively, denote the number of clusters that are assigned only the integer " 1 ," only the integer " 2 ," only the integer " 3 ," both the integers "1" and " 2 ," both the integers " 2 " and " 3 ," and both the integers "1" and "3." Since the interleaving on $G$ is an MCI, any $m$ nonoverlapping clusters are assigned at least $K=3$ distinct integers. Therefore, $x+y+a \leq m-1, y+z+b \leq m-1$, $z+x+c \leq m-1$. So $2 x+2 y+2 z+a+b+c \leq 3 m-3$ and hence, $x+y+z+a+b+c \leq 3 m-3-(x+y+z)$. Since $x+y+z \geq 1, t=x+y+z+a+b+c$, and $n \leq 2 t+2$, we get

$$
\begin{aligned}
n & \leq 2(x+y+z+a+b+c+1) \\
& \leq 2[3 m-3-(x+y+z)+1] \\
& \leq 6 m-6=(N-1)[(m-1) N-1]+2 .
\end{aligned}
$$

Therefore this lemma is proved.

With Lemmas 2 and 3 proved, we see that Theorem 1 becomes a natural conclusion.

\section{OPtimal CONSTRUCTION FOR MCI ON PATHS With CONSTRAINTS $L=2$ AND $K=3$}

In this section, we present a construction for MCI on paths whose orders attain the upper bound of Theorem 1, therefore proving the exactness of that bound. The construction is shown as the following algorithm.

Algorithm 1: $M C I$ on the longest path with constraints $L=2$ and $K=3$

Input: Parameters $N, K, m$ and $L$, where $N \geq 3, K=3$, $m \geq 2$ and $L=2$. A path $G=(V, E)$ of $n=(N-1)[(m-1) N-1]+2$ vertices.

Output: An MCI on $G$.

\section{Algorithm:}

Let $H=\left(V_{H}, E_{H}\right)$ be a graph with parallel edges. The vertex set of $H, V_{H}$, is $\left\{u_{1}, u_{2}, \ldots, u_{N}\right\}$. For any two vertices $u_{i}$ and $u_{j}(i \neq j)$, there are $2 m-3$ edges between them if $2 \leq i=$ $j+1 \leq N-1$ or $2 \leq j=i+1 \leq N-1$, and there are $2 m-2$ edges between them otherwise. There is no loop in $H$. (Therefore $H$ has exactly $n-1$ edges.)

Find a walk in $H, u_{k_{1}} \rightarrow u_{k_{2}} \rightarrow \cdots \rightarrow u_{k_{n}}$, that satisfies the following two requirements: 1 ) the walk starts with $u_{1}$ and ends with $u_{N-1}$-namely, $u_{k_{1}}=u_{1}$ and $u_{k_{n}}=u_{N-1}$ - and passes every edge in $H$ exactly once; 2 ) for any two vertices of $H$, the walk passes all the edges between them consecutively.

For $i=1,2, \ldots, n$, assign the integer " $k_{i}$ " to the vertex $v_{i}$ in $G$, and we get an MCI on $G$.

Here is an example of the above algorithm.

Example 2: Assume $G=(V, E)$ is a path of $n=11$ vertices, and the parameters are $N=4, K=3, m=2$, and $L=2$. Therefore $n=(N-1)[(m-1) N-1]+2$. Algorithm 1 constructs a graph $H=\left(V_{H}, E_{H}\right)$, which is shown in 
(a)

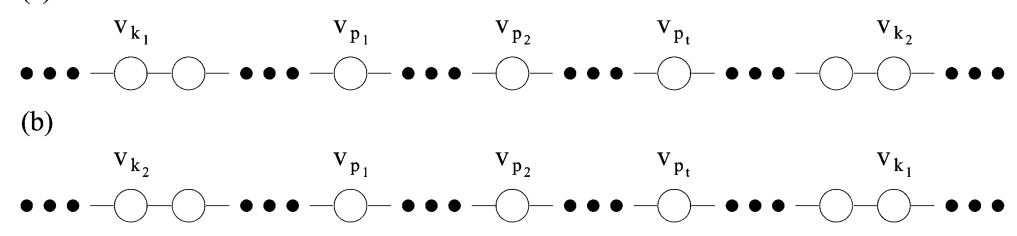

Fig. 8. (a) Case 1: $k_{1}<k_{2}$. (b) Case 2: $k_{2}<k_{1}$.

$$
\mathrm{n}=11, \quad \mathrm{~N}=4, \quad \mathrm{~K}=3, \quad \mathrm{~m}=2, \quad \mathrm{~L}=2
$$
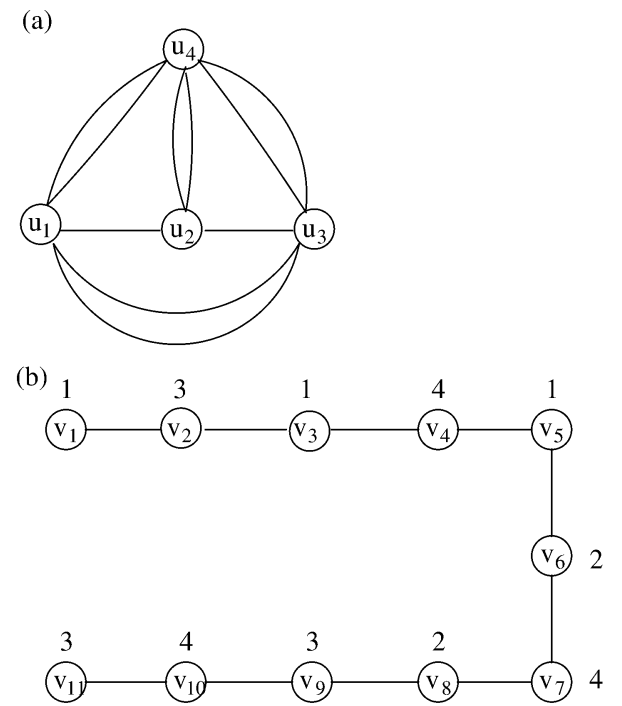

Fig. 9. (a) The graph $H=\left(V_{H}, E_{H}\right)$. (b) MCI on the path $G=(V, E)$.

Fig. 9(a). The walk in $H, u_{k_{1}} \rightarrow u_{k_{2}} \rightarrow \cdots \rightarrow u_{k_{n}}$, can be easily found. For example, we can let the walk be

$$
u_{1} \rightarrow u_{3} \rightarrow u_{1} \rightarrow u_{4} \rightarrow u_{1} \rightarrow u_{2} \rightarrow u_{4} \rightarrow u_{2} \rightarrow u_{3} \rightarrow u_{4} \rightarrow u_{3}
$$

Corresponding to that walk, we get the interleaving on $G$ as shown in Fig. 9(b). It can be easily verified that the interleaving is indeed an MCI.

Theorem 2: Algorithm 1 correctly outputs an MCI on the path $G$.

Proof of Theorem 2: The interleaving on $G$ that Algorithm 1 outputs corresponds to a walk in the graph $H=\left(V_{H}, E_{H}\right)$. The $N$ vertices of $H$ correspond to the $N$ integers interleaved on $G$. It is not difficult to realize that the walk in $H$ satisfying its two requirements indeed exists. For any two vertices $u_{i}$ and $u_{j}$ in $H$, there are at most $2 m-2$ edges between them, which are passed consecutively by the walk. So $G$ has at most $2 m-2$ edges whose endpoints are assigned the integers $i$ and $j$, and those edges are consecutive in $G$. So $G$ has at most $m-1$ nonoverlapping clusters that are assigned only integers $i$ and $j$. Now it is simple to see that the interleaving on $G$ is an MCI.

Algorithm 1 is optimal in the sense that it produces MCI for the longest path on which MCI exists. It is clear that the algorithm can be modified easily to produce MCI for shorter paths as well - the method is to find shorter walks in the auxiliary graph $H=\left(V_{H}, E_{H}\right)$. We skip the details for simplicity. By Theorems 1 and 2, we find the exact condition for MCI's existence when $L=2$ and $K=3$, as presented in the following theorem. (a)

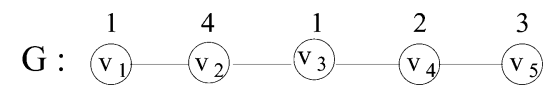

(b)

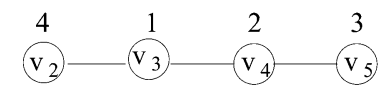

(c)

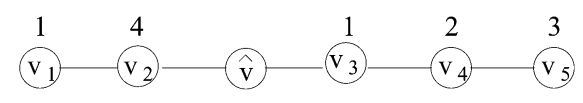

(d)

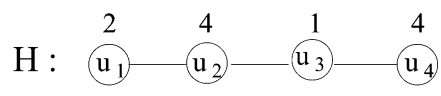

(e)

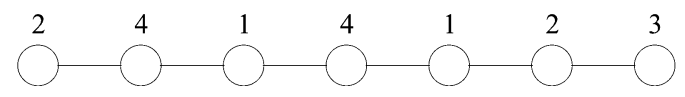

Fig. 10. Illustrations of three operations on paths.

Theorem 3: When $L=2$ and $K=3$, there exists an MCI on a path of $n$ vertices if and only if $n \leq(N-1)[(m-1) N-1]+2$.

\section{MCI ON PATHS With Constraints $K=L+1$}

In this section, we study the MCI problem for paths with a more general constraint: $K=L+1$.

We define three operations on paths_-"remove a vertex," "insert a vertex," and "combine two paths." Let $G$ be a path of $n$ vertices: $\left(v_{1}, v_{2}, \ldots, v_{n}\right)$. By "removing the vertex $v_{i}$ " from $G(1 \leq i \leq n)$, we get a new path $\left(v_{1}, v_{2}, \ldots, v_{i-1}, v_{i+1}, \ldots, v_{n}\right)$. By "inserting a vertex $\hat{v}$ " in front of the vertex $v_{i}$ in $G(1 \leq i \leq n)$, we get a new path $\left(v_{1}, v_{2}, \ldots v_{i-1}, \hat{v}, v_{i}, \ldots, v_{n}\right)$. Let $H$ be a path of $n^{\prime}$ vertices: $\left(u_{1}, u_{2}, \ldots, u_{n^{\prime}}\right)$. Assume for $1 \leq i \leq n, v_{i}$ is assigned the integer $c\left(v_{i}\right)$; and assume for $1 \leq i \leq n^{\prime}, u_{i}$ is assigned the integer $c\left(u_{i}\right)$. Also, let $l$ be a positive integer between 1 and $\min \left(n, n^{\prime}\right)$, and assume for $1 \leq i \leq l, c\left(v_{i}\right)=c\left(u_{n^{\prime}-l+i}\right)$. Then by saying "combining $H$ with $G$ such that the last $l$ vertices of $H$ overlap the first $l$ vertices of $G$," we mean to construct a path of $n^{\prime}+n-l$ vertices whose assigned integers are in the form of

$$
\left[c\left(u_{1}\right), c\left(u_{2}\right), \ldots, c\left(u_{n^{\prime}}\right), c\left(v_{l+1}\right), c\left(v_{l+2}\right), \ldots, c\left(v_{n}\right)\right]
$$

which is the same as

$$
\left[c\left(u_{1}\right), c\left(u_{2}\right), \ldots, c\left(u_{n^{\prime}-l}\right), c\left(v_{1}\right), c\left(v_{2}\right), \ldots, c\left(v_{n}\right)\right] .
$$

The following are examples of the three operations.

Example 3: Let $G$ be the path shown in Fig. 10(a). By removing the vertex $v_{1}$ from $G$, we get the path shown in Fig. 10(b). By inserting a vertex $\hat{v}$ in front of the vertex $v_{3}$ in $G$ (or equivalently, behind the vertex $v_{2}$ in $G$, or between the vertex $v_{2}$ and $v_{3}$ in $G$ ), we get the path shown in Fig. 10(c). 
Let $H$ be the path shown in Fig. 10(d). By combining $H$ with $G$ such that the last two vertices of $H$ overlap the first two vertices of $G$, we get the path shown in Fig. 10(e).

Now we present an algorithm which computes an MCI on a path while $K=L+1$. Being different from Algorithm 1, in this algorithm the order of the path is not preset. Instead, the algorithm tries to find a long path on which MCI exists (the longer, the better), and computes an MCI for it. Thus, the output of this algorithm not only provides an MCI solution, but also gives a lower bound for the maximum order of the path on which MCI exists.

Algorithm 2: $M C I$ on a path with the constraint $K=L+1$

Input: Parameters $N, K, m$ and $L$, where $N \geq K=L+1 \geq 3$ and $m \geq 2$.

Output: An MCI on a path $G=(V, E)$.

Algorithm:

1. If $L=2$, then let $G=(V, E)$ be a path of

$$
(N-1)[(m-1) N-1]+2
$$

vertices, and use Algorithm 1 to find an MCI on $G$. Output $G$ and the MCI on it, then exit. (So Step 2 and

Step 3 will be executed only if $L \geq 3$.)

2. for $i=L+1$ to $N$ do

$\left\{\right.$ Find a path $B_{i}$ (the longer, the better) that satisfies the following three conditions:

(1) Each vertex of $B_{i}$ is assigned an integer in $\{1,2, \ldots, i-1\}$, namely, there is an interleaving of the integers in $\{1,2, \ldots, i-1\}$ on $B_{i}$;

(2) Any $m$ nonoverlapping connected subgraphs of $B_{i}$, each of which is of order $L-1$, are assigned at least $L$ distinct integers;

(3) If $i>L+1$, then for $j=1$ to $L-1$, the $j$ th last vertex of $B_{i}$ is assigned the same integer as the $(L-j)$ th vertex of $A_{i-1}$.

To find the path $B_{i}$, (recursively) call Algorithm 2 in the following way: when calling Algorithm 2, replace the inputs of the algorithm- $N, K, m$, and $L-$ respectively, with $i-1, L, m$, and $L-1$; then let the output of Algorithm 2 (which is a path with an interleaving on it) be the path $B_{i}$.

Scan the vertices in $B_{i}$ backward (from the last vertex to the first vertex), and insert a new vertex after every $L-1$ vertices in $B_{i}$. (In other words, if the vertices in $B_{i}$ are $u_{1}, u_{2}, \ldots, u_{\hat{n}}$, then after inserting vertices into $B_{i}$ in the way described above, we get a new path of

$$
\hat{n}+\left\lfloor\frac{\hat{n}}{L-1}\right\rfloor
$$

vertices; and if we look at the new path in the reverse order-from the last vertex to the first vertex-then the path is of the form $\left(u_{\hat{n}}, u_{\hat{n}-1}, \ldots, u_{\hat{n}+1-(L-1)}\right.$, a new vertex, $u_{\hat{n}-(L-1)}, u_{\hat{n}-(L-1)-1}, \ldots, u_{\hat{n}+1-2(L-1)}$, a new vertex, $u_{\hat{n}-2(L-1)}, u_{\hat{n}-2(L-1)-1}, \ldots, u_{\hat{n}+1-3(L-1)}$, a new vertex, ...). In this new path, every cluster of order $L$ contains exactly one newly inserted vertex.)

Assign the integer " $i$ " to every newly inserted vertex in the new path, and denote this new path by " $A_{i}$."

\}

3. Obtain a new path by combining the paths $A_{N}, A_{N-1}, \ldots$,
$A_{L+1}$ in the following way: combine $A_{N}$ with $A_{N-1}$, combine $A_{N-1}$ with $A_{N-2}, \ldots$, and combine $A_{L+2}$ with $A_{L+1}$ such that the last $L-1$ vertices of $A_{N}$ overlap the first $L-1$ vertices of $A_{N-1}$, the last $L-1$ vertices of $A_{N-1}$ overlap the first $L-1$ vertices of $A_{N-2}, \ldots$, and the last $L-1$ vertices of $A_{L+2}$ overlap the first $L-1$ vertices of $A_{L+1}$. (In other words, if we denote the number of vertices in $A_{i}$ by $l_{i}$, for $L+1 \leq i \leq N$, then the new path we get has

$$
\sum_{i=L+1}^{N} l_{i}-(L-1)(N-L-1)
$$

vertices.) Let this new path be $G=(V, E)$. Output $G$ and the interleaving (which is an MCI) on it, then exit.

The following is an example of Algorithm 2.

Example 4: In this example, the input parameters for Algorithm 2 are $N=6, K=4, m=2$, and $L=3$. That is, we use Algorithm 2 to compute a path that is the longer the better and interleaves 6 integers on it, such that in the path, any 2 nonoverlapping clusters are assigned at least 4 distinct integers.

Algorithm 2 first computes a path $B_{4}$ that satisfies the following two conditions: 1 ) each vertex of $B_{4}$ is assigned an integer in $\{1,2,3\} ; 2)$ any $m=2$ nonoverlapping connected subgraphs of $B_{4}$ of order $L-1=2$ are assigned at least $L=3$ distinct integers. To compute $B_{4}$, Algorithm 2 calls itself in a recursive way, by setting the inputs of the algorithm-N, $K, m$, and $L$ - to be 3,3,2, and 2; during that call, it uses Algorithm 1 to compute $B_{4}$. There is more than one possible outcome of Algorithm 1; WLOG, let us assume the output here is that $B_{4}$ is assigned integers in the form of $[1,3,1,2,3,2]$. The path $B_{4}$ is shown in Fig. 11(a).

Algorithm 2 then scans $B_{4}$ backward, inserts a new vertex into $B_{4}$ after every $L-1=2$ vertices, and assigns the integer " 4 " to every newly inserted vertex. As a result, we get a path whose assigned integers are in the form of $[4,1,3,4,1,2,4,3,2]$. We call this new path $A_{4}$. $A_{4}$ is shown in Fig. 11(b).

Algorithm 2 then computes a path $B_{5}$ that satisfies the following three conditions: 1 ) each vertex of $B_{5}$ is assigned an integer in $\{1,2,3,4\} ; 2)$ any $m=2$ nonoverlapping connected subgraphs of $B_{5}$ of order $L-1=2$ are assigned at least $L=3$ distinct integers; 3 ) the last vertex of $B_{5}$ is assigned the same integer as the second vertex of $A_{4}$ (which is the integer " 1 "), and the second last vertex of $B_{5}$ is assigned the same integer as the first vertex of $A_{4}$ (which is the integer "4").

Algorithm 2 computes $B_{5}$ by once again calling itself. Algorithm 2 can use the following method to find a path that satisfies all the above three conditions. First, use Algorithm 1 to find a path that satisfies the first two conditions, which is easy, and call this path $C_{5}$. All the integers assigned to $C_{5}$ are in the set $\{1,2,3,4\}$; and from Algorithm 1, it is simple to see that the last two vertices in $C_{5}$ are assigned two different integers. (Note that the first two vertices in $A_{4}$ are also assigned two different integers.) So by permuting the names of the integers assigned to $C_{5}$, we can get a path that satisfies not only the first two conditions but also the third condition. Call this path $B_{5}$. There is more than one possible result of $B_{5}$. WLOG, we assume the integers assigned to $B_{5}$ are in the form of

$$
[3,4,3,1,3,2,4,2,1,4,1] \text {. }
$$


$\mathrm{N}=6, \quad \mathrm{~K}=4, \quad \mathrm{~m}=2, \quad \mathrm{~L}=3$

(a) $\mathrm{B}_{4}$

$$
\text { (1) } 3-1-2-3
$$

(b) $\mathrm{A}_{4}$

$$
\text { (4)-(1)-(3)-(1)-3-2) }
$$

(c) $\mathrm{B}_{5}$

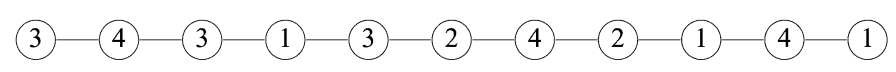

(d) $\mathrm{A}_{5}$

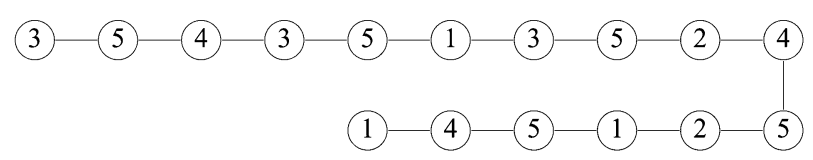

(e) $\mathrm{B}_{6}$

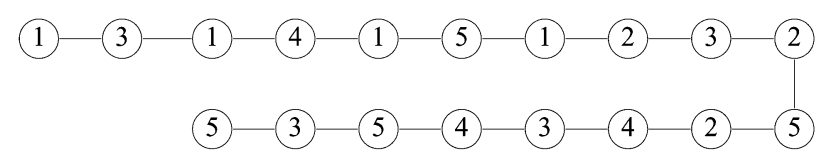

(f) $\mathrm{A}_{6}$

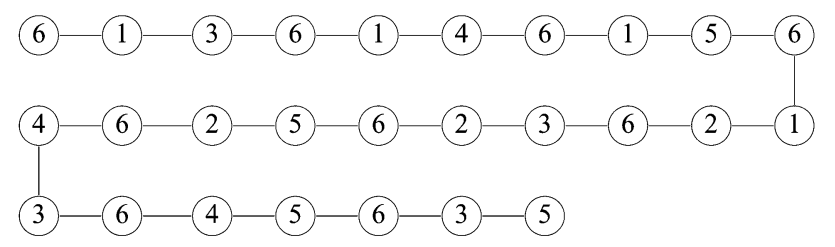

(g) $\mathrm{G}=(\mathrm{V}, \mathrm{E})$

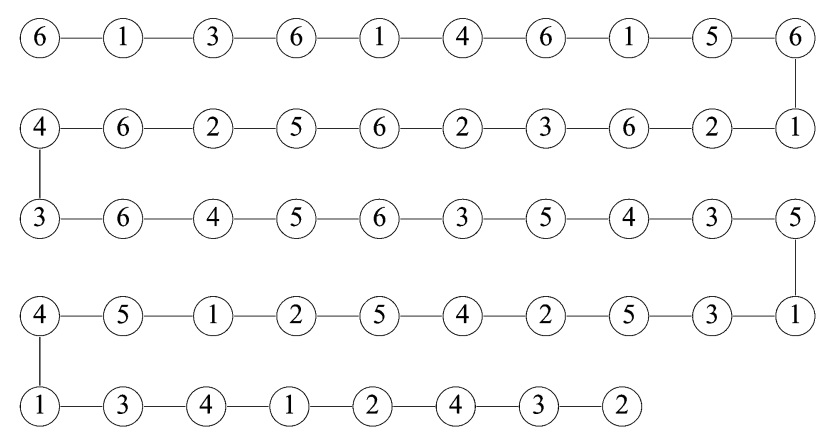

Fig. 11. An example of Algorithm 2.

$B_{5}$ is shown in Fig. 11(c). Then Algorithm 2 inserts vertices into $B_{5}$ and gets a new path $A_{5}$, whose assigned integers are in the form of

$[3,5,4,3,5,1,3,5,2,4,5,2,1,5,4,1]$.

$A_{5}$ is shown in Fig. 11(d).

Next, Algorithm 2 computes a path $B_{6}$, by calling itself again. WLOG, we assume the integers assigned to $B_{6}$ are in the form of
$B_{6}$ is shown in Fig. 11(e). Then Algorithm 2 inserts vertices into $B_{6}$ and gets a new path $A_{6}$, whose assigned integers are in the form of

$[6,1,3,6,1,4,6,1,5,6,1,2,6,3,2,6,5,2,6,4,3,6,4,5,6,3,5]$. $A_{6}$ is shown in Fig. 11(f).

Finally, Algorithm 2 combines $A_{6}, A_{5}$, and $A_{4}$ such that the last $L-1=2$ vertices of $A_{6}$ overlap the first two vertices of $A_{5}$, and the last $L-1=2$ vertices of $A_{5}$ overlap the first two vertices of $A_{4}$. As a result, we get a path $G=(V, E)$ of 48 vertices which is assigned the integers 


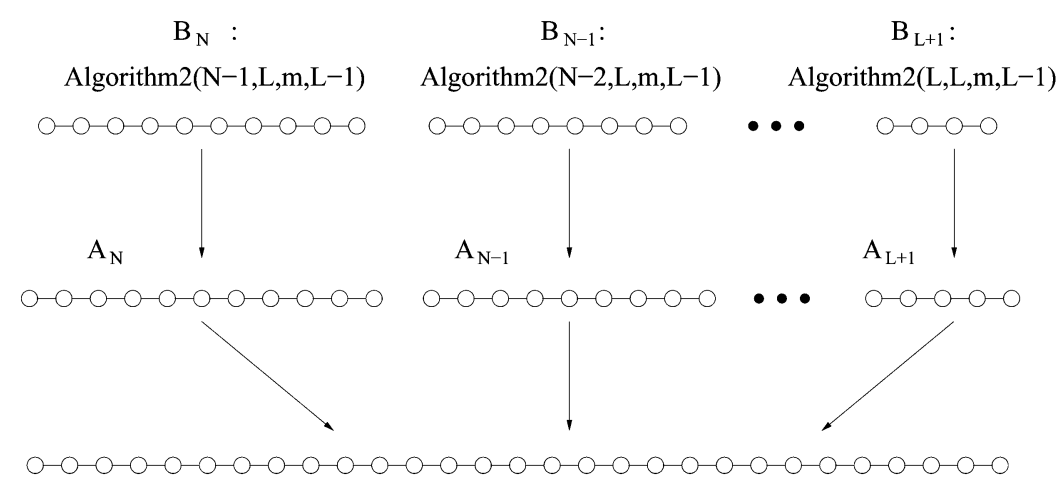

Algorithm2(N,L+1,m,L)

Fig. 12. Algorithm 2 has four input parameters: $N, K, m$, and $L$. Let us use "Algorithm $2(a, b, c, d)$ " to denote the path output by Algorithm 2 when $N=a$, $K=b, m=c$, and $L=d$. The final output of Algorithm 2-Algorithm2 $(N, L+1, m, L)$-is obtained by combining the paths $A_{N}, A_{N-1}, \ldots, A_{L+1}$, while $A_{i}$ (for $i=N, N-1, \ldots, L+1$ ) is obtained by inserting vertices into the path $B_{i} . B_{i}$ is an output of Algorithm 2 as well, which is a path with an interleaving of $i-1$ different integers; specifically, $B_{i}$ is Algorithm2 $(i-1, L, m, L-1)$. So from this figure, we can see the recursive structure of Algorithm 2 , and the "hierarchical-chain structure" of its output.

$G$ is shown in Fig. 11(g). This is the output of Algorithm 2. It can be verified that the interleaving on $G$ is indeed an MCI.

The path output by Algorithm 2 is a chain of the subpaths $A_{L+1}, A_{L+2}, \ldots, A_{N}$. The interleavings on those paths use more and more integers, and those subpaths are of increasing orders. In that sense, they form a "hierarchy." Each subpath $A_{i}$ is derived from a path $B_{i}$, and $B_{i}$ is a chain of some shorter subpaths; then, each of the subpaths that constitute $B_{i}$ is derived through the chaining of some even shorter subpaths, and so on. That is another "hierarchy." Therefore, we say that the path output by Algorithm 2 has a "hierarchical-chain structure." (See Fig. 12 for an illustration.)

The complexity of Algorithm 2 is dominated by the total number of vertices generated during the running of Algorithm 2. That number is greater than the order of the final output path $G=(V, E)$ (except when $L=2)$, because when Algorithm 2 is combining paths, there are overlapping vertices. However, we can show that the total number of vertices generated is less than twice the order of $G=(V, E)$. A proof of this claim is presented in Appendix I.

In the following we prove the correctness of Algorithm 2.

Theorem 4: Algorithm 2 is correct.

Proof of Theorem 4: We will prove this theorem by induction. If $L=2$, then Algorithm 2 uses Algorithm 1 to compute the MCI-so the result is clearly correct. Also, we notice that for any MCI output by Algorithm 1, any two adjacent vertices are assigned different integers. We use those two facts as the base case.

Let $I$ be an integer such that $2<I \leq L$. Let us assume the following statement is true: if we replace the inputs of Algorithm 2-parameters $N, K, m$, and $L$-with any other set of valid inputs $\hat{N}, \hat{K}=i+1, \hat{m}$, and $i$ such that $2 \leq i<I$, Algorithm 2 will correctly output an MCI on a path; and in that MCI, any $i$ consecutive vertices are assigned $i$ different integers. (That is our induction assumption.)

Now let us replace the inputs of Algorithm 2-parameters $N, K, m$, and $L$-with a set of valid inputs $N^{\prime}, K^{\prime}=$ $I+1, m^{\prime}$, and $I$. Then Algorithm 2 needs to compute (in its Step 2) $N^{\prime}-I$ paths: $B_{I+1}, B_{I+2}, \ldots, B_{N^{\prime}}$. For
$I+1 \leq j \leq N^{\prime}, B_{j}$ is (recursively) computed by calling Algorithm 2. The interleaving on $B_{j}$ is in fact an MCI where the order of each cluster is $I-1$-so by the induction assumption, Algorithm 2 will correctly output the interleaving on $B_{j} . B_{j}$ is assigned the integers in $\{1,2, \ldots, j-1\}$; and by the induction assumption, any $I-1$ consecutive vertices in $B_{j}$ are assigned $I-1$ different integers.

The path $A_{I+1}$ is constructed by inserting vertices into $B_{I+1}$ such that any $I$ consecutive vertices in $A_{I+1}$ contain exactly one newly inserted vertex, and all the newly inserted vertices are assigned the integer " $I+1$." So any $I$ consecutive vertices in $A_{I+1}$ are assigned $I$ different integers. Therefore, it is always feasible to adjust the interleaving on $B_{I+2}$ to make the last $I-1$ vertices of $B_{I+2}$ be assigned the same integers as the first $I-1$ vertices of $A_{I+1}$. Noticing that the last $I-1$ vertices of $B_{I+2}$ are assigned the same integers as the last $I-1$ vertices of $A_{I+2}$, we see that $A_{I+2}$ and $A_{I+1}$ can be successfully combined with $I-1$ overlapping vertices by Algorithm 2. Similarly, for $I+3 \leq t \leq$ $N^{\prime}, A_{t}$ and $A_{t-1}$ can be successfully combined by Algorithm 2 ; and for $I+2 \leq t \leq N^{\prime}$, any $I$ consecutive vertices in $A_{t}$ are assigned $I$ different integers.

Algorithm 2 uses $G$ to denote the path got by combining $A_{L+1}, A_{L+2}, \ldots, A_{N}$. For our discussion here, $L$ and $N$ should, respectively, be replaced by $I$ and $N^{\prime}$. Clearly, any $I$ consecutive vertices in $G$ are also $I$ consecutive vertices in $A_{j}$ for some $j\left(I+1 \leq j \leq N^{\prime}\right)$, therefore are assigned $I$ different integers. And for any $m^{\prime}$ nonoverlapping connected subgraphs of order $I$ in $G$, either all of them are contained in $A_{j}$ for some $j\left(I+1 \leq j \leq N^{\prime}\right)$, or one of them is contained in $A_{j^{\prime}}$ and another of them is contained in $A_{j^{\prime \prime}}$ for some $j^{\prime \prime} \neq j^{\prime}\left(I+1 \leq j^{\prime} \neq j^{\prime \prime} \leq N^{\prime}\right)$. In the former case, by removing those vertices that are assigned the integer " $j$ " in those $m^{\prime}$ subgraphs, we get $m^{\prime}$ nonoverlapping connected subgraphs in $B_{j}$ each of which contains $I-1$ vertices, which in total are assigned at least $I$ different integers not including " $j$ "the $m^{\prime}$ subgraphs in $G$ (which are also in $A_{j}$ ) are assigned at least $I+1$ different integers. In the latter case, WLOG, let us say $j^{\prime}<j^{\prime \prime}$. Then the subgraph in $A_{j^{\prime}}$ is assigned $I$ different integers not including " $j$ ", " and the subgraph in $A_{j^{\prime \prime}}$ is assigned an integer " $j$ " " - so the $m$ ' subgraphs in $G$ are assigned at least 
$I+1$ different integers in total. Therefore, the interleaving on $G$ is an MCI (with parameters $N^{\prime}, K^{\prime}, m^{\prime}$, and $I$ ). So the induction assumption also holds when $i=I$.

Algorithm 2 computes the result for the original problem by recursively calling itself. By the above induction, every intermediate time Algorithm 2 is called, the output is correct. So the final output of Algorithm 2 is also correct.

The maximum order of a path for which MCI exists increases when $N$-the number of interleaved integers-increases. The performance of Algorithm 2 can be evaluated by the difference between the order of the path output by Algorithm 2 and the maximum order of a path for which MCI exists. We are interested in how the difference behaves when $N$ increases.

Theorem 5: Fix the values of $K, m$, and $L$, where $K=$ $L+1 \geq 3$ and $m \geq 2$, and let $N$ be a variable $(N \geq K)$. Then the longest path for which MCI exists has

$$
\frac{m-1}{(L-1) !} N^{L}+O\left(N^{L-1}\right)
$$

vertices. And the path output by Algorithm 2 also has

$$
\frac{m-1}{(L-1) !} N^{L}+O\left(N^{L-1}\right)
$$

vertices.

Proof of Theorem 5: Let $G=(V, E)$ be a path of $n$ vertices with an MCI on it. Then by Proposition 1

$$
n \leq(m-1) L\left(\begin{array}{c}
N \\
L
\end{array}\right)+(L-1) \text {. }
$$

So

$$
n \leq \frac{m-1}{(L-1) !} N^{L}+O\left(N^{L-1}\right) .
$$

When $L=2$, Algorithm 2 outputs a path of $(N-1)[(m-$ 1) $N-1]+2$ vertices. When $L \geq 3$, to get the output, Algorithm 2 needs to construct the paths $A_{L+1}, A_{L+2}, \ldots, A_{N}$; and for $L+1 \leq i \leq N, A_{i}$ is got by inserting vertices into the path $B_{i}$. $B_{i}$ is again an output of Algorithm 2, which is assigned $i-1$ distinct integers, and in which a considered "cluster" is of order $L-1$. Let us use $F(N, m, L)$ to denote the number of vertices in the path output by Algorithm 2, and use $A(i, m, L)$ to denote the number of vertices in the path $A_{i}$. Then based on the above observed relations, we get the following three equations:

1) $F(N, m, 2)=(N-1)[(m-1) N-1]+2$;

2) when $L \geq 3$

$$
F(N, m, L)=\sum_{i=L+1}^{N} A(i, m, L)-(N-L-1)(L-1)
$$

3) when $i \geq L+1 \geq 4$

$$
A(i, m, L)=\left\lfloor\frac{L}{L-1} \cdot F(i-1, m, L-1)\right\rfloor .
$$

(Note that $F(i-1, m, L-1)$ is the number of vertices in the path $B_{i}$.)

\begin{tabular}{|c|c|c|c|}
\hline & \multicolumn{3}{|c|}{$m=2$ and $L=3$} \\
\hline$N$ & $\begin{array}{l}\text { Output of } \\
\text { Algorithm } 2(n)\end{array}$ & $\begin{array}{c}\text { Upper } \\
\text { bound }\left(U_{\text {bound }}\right)\end{array}$ & $\begin{array}{r}\text { Relative } \\
\text { difference }\end{array}$ \\
\hline 10 & 312 & 362 & 0.1381 \\
\hline 20 & 3177 & 3422 & 0.0716 \\
\hline 50 & 57072 & 58802 & 0.0294 \\
\hline 100 & 477897 & 485102 & 0.0149 \\
\hline 150 & 1637472 & 1653902 & 0.0099 \\
\hline \multirow[t]{2}{*}{200} & 3910797 & 3940202 & 0.0075 \\
\hline & \multicolumn{3}{|c|}{$m=2$ and $L=5$} \\
\hline$N$ & $\begin{array}{l}\text { Output of } \\
\text { Algorithm } 2(n)\end{array}$ & $\begin{array}{c}\text { Upper } \\
\left.\text { bound ( } U_{\text {bound }}\right)\end{array}$ & $\begin{array}{r}\text { Relative } \\
\text { difference }\end{array}$ \\
\hline 10 & 930 & 1264 & 0.2642 \\
\hline 20 & 68265 & 77524 & 0.1194 \\
\hline 50 & 10081020 & 10593804 & 0.0484 \\
\hline 100 & 367196445 & 376437604 & 0.0245 \\
\hline 150 & $2.9093 \times 10^{9}$ & $2.9580 \times 10^{9}$ & 0.0165 \\
\hline \multirow[t]{2}{*}{200} & $1.2521 \times 10^{10}$ & $1.2678 \times 10^{10}$ & 0.0124 \\
\hline & \multicolumn{3}{|c|}{$m=5$ and $L=3$} \\
\hline$N$ & $\begin{array}{l}\text { Output of } \\
\text { Algorithm } 2(n)\end{array}$ & $\begin{array}{c}\text { Upper } \\
\text { bound }\left(U_{\text {bound }}\right)\end{array}$ & $\begin{array}{r}\text { Relative } \\
\text { difference }\end{array}$ \\
\hline 10 & 1383 & 1442 & 0.0409 \\
\hline 20 & 13428 & 13682 & 0.0186 \\
\hline 50 & 233463 & 235202 & 0.0074 \\
\hline 100 & 1933188 & 1940402 & 0.0037 \\
\hline 150 & 6599163 & 6615602 & 0.0025 \\
\hline \multirow[t]{2}{*}{200} & 15731388 & 15760802 & 0.0019 \\
\hline & \multicolumn{3}{|c|}{$m=5$ and $L=5$} \\
\hline$N$ & $\begin{array}{l}\text { Output of } \\
\text { Algorithm } 2(n)\end{array}$ & $\begin{array}{c}\text { Upper } \\
\text { bound }\left(U_{\text {bound }}\right)\end{array}$ & $\begin{array}{r}\text { Relative } \\
\text { difference }\end{array}$ \\
\hline 10 & 4395 & 5044 & 0.1287 \\
\hline 20 & 298785 & 310084 & 0.0364 \\
\hline 50 & 41846205 & 42375204 & 0.0125 \\
\hline 100 & $1.4964 \times 10^{9}$ & $1.5058 \times 10^{9}$ & 0.0062 \\
\hline 150 & $1.1783 \times 10^{10}$ & $1.1832 \times 10^{10}$ & 0.0041 \\
\hline 200 & $5.0556 \times 10^{10}$ & $5.0713 \times 10^{10}$ & 0.0031 \\
\hline
\end{tabular}

By solving the above equations, we get

as claimed.
TABLE I

COMPARISON BETWEEN THE ORDER OF THE PATH OUTPUT BY ALGORITHM 2 AND AN UPPER BOUND, AND THEIR RELATIVE DIFFERENCE

Theorem 5 shows that the path output by Algorithm 2 is asymptotically as long as the longest path for which MCI exists. Additionally, the orders of those two paths have the same highest degree term (in $N$ ).

We conclude with some numerical results. In Table I, the order of the path output by Algorithm $2-n-$ is compared with the upper bound of Proposition $1-U_{\text {bound }}-$ for four different sets of parameters $m$ and $L$, with $K=L+1$ throughout. The "relative difference" in Table I is defined as

$$
\frac{U_{\text {bound }}-n}{U_{\text {bound }}}=1-\frac{n}{U_{\text {bound }}} \text {. }
$$

Theorem 5 shows that this relative difference approaches 0 as $N \rightarrow \infty$.

\section{MCI ON CYCLES}

In this section, we extend our results on MCI from paths to cycles, for the case of " $L=2$ and $K=3$." The analysis for the two kinds of graphs bears similarity; but the "circular" structure of the cycle leads to certain differences sometimes.

Let $G=(V, E)$ be a cycle. The following notations will be used throughout this section. We denote the $n$ vertices in $G=(V, E)$ by $v_{1}, v_{2}, \ldots, v_{n}$. For $2 \leq i \leq n-1$, the two vertices adjacent to $v_{i}$ are $v_{i-1}$ and $v_{i+1}$. Vertex $v_{1}$ and $v_{n}$ are 
adjacent to each other. A connected subgraph of $G$ induced by vertices $v_{i}, v_{i+1}, \ldots, v_{j}$ is denoted by $\left(v_{i}, v_{i+1}, \ldots, v_{j}\right)$. If a set of integers are interleaved on $G$, then $c\left(v_{i}\right)$ denotes the integer assigned to vertex $v_{i}$.

Lemma 4: Let the values of $N, K, m$, and $L$ be fixed, where $N \geq 4, K=3, m \geq 2$, and $L=2$. Let $n_{\max }$ denote the maximum value of $n$ such that an MCI exists on a cycle of $n$ vertices. Then in any MCI on a cycle of $n_{\max }$ vertices, no two adjacent vertices are assigned the same integer.

The proof of Lemma 4 is skipped because it is very similar to that of Lemma 1.

Lemma 5: Let the values of $N, K, m$, and $L$ be fixed, where $N \geq 4, K=3, m \geq 2$, and $L=2$. Let $n_{\max }$ denote the maximum value of $n$ such that an MCI exists on a cycle of $n$ vertices. Then $n_{\max } \leq(N-1)[(m-1) N-1]$.

Proof of Lemma 5: This lemma can be proved in the same way as the proof for Lemma 2, except for a few small differences. For simplicity, we just point out those differences here, and skip the rest of the proof.

The first difference is that due to the "circular" topology of the cycle $G$, the specific way to color the vertices of $G$ with the red, yellow, and green colors should be modified to be the following: "Step 1) for $1 \leq i \leq n_{\max }$, if the two vertices adjacent to $v_{i}$ are assigned the same integer, then we color $v_{i}$ with the red color; Step 2) for $1 \leq i \leq n_{\max }$, we color $v_{i}$ with the yellow color if $v_{i}$ is not colored red and there exists $j$ such that these four conditions are satisfied: 1) $j \neq i, 2) v_{j}$ is not colored red, (3) $\left.c\left(v_{j}\right)=c\left(v_{i}\right), 3\right)$ the following vertices between $v_{j}$ and $v_{i}-v_{j+1}, v_{j+2}, \ldots, v_{i-1}$ (note that if a lower index exceeds $n_{\max }$, it is subtracted by $n_{\max }$, so that the lower index is always between 1 and $n_{\max }$ ) - are all colored red; Step 3) for $1 \leq i \leq n_{\max }$, if $v_{i}$ is neither colored red nor colored yellow, then we color $v_{i}$ with the green color."

The second difference is that compared to paths, for cycles there are two extra cases to consider in the proof:

Case 1: all the vertices in the cycle $G$ are red. If that is true, then $G$ must have been assigned only two distinct integers, which implies that $G$ contains less than $m L=2 m<(N-1)[(m-1) N-1]$ vertices (since we assume the interleaving on $G$ is an MCI);

Case 2: there is no green vertex in $G$, and all the yellow vertices are assigned the same integer - say it is integer " $i$." If that is true, then the integers on $G$ must look like the following:

$$
\begin{aligned}
&\{i, a, i, a, \ldots, i, a, i, b, i, b, \ldots, i \\
&b, \ldots \ldots, i, c, i, c, \ldots, i, c\} .
\end{aligned}
$$

For any $j \neq i(1 \leq j \leq N)$, there are at most $2 m-2$ edges in $G$ whose endpoints are assigned $i$ and $j$, respectively (because the interleaving on $G$ is an MCI). So the order of $G$ (which equals the number of edges in $G$ ) is at most

$$
(N-1)(2 m-2)<(N-1)[(m-1) N-1] .
$$

Lemma 6: Let the values of $N, K, m$, and $L$ be fixed, where $N=3, K=3, m \geq 2$, and $L=2$. Let $n_{\max }$ denote the maximum value of $n$ such that an MCI exists on a cycle of $n$ vertices. Then $n_{\max } \leq(N-1)[(m-1) N-1]$.

Proof of Lemma 6: Let $G=(V, E)$ be a cycle of $n_{\max }$ vertices that has an MCI on it. We need to show that $n_{\max } \leq$ $(N-1)[(m-1) N-1]$. It is simple to see that $G$ is assigned $N=$ 3 distinct integers. If in the MCI on $G$, no two adjacent vertices are assigned the same integer, then with the same argument as in the proof of Lemma 5, it can be shown that $n_{\max } \leq(N-$ $1)[(m-1) N-1]$. Now assume there are two adjacent vertices in $G$ that are assigned the same integer. Then there are three possible cases.

Case 1: $n_{\max }$ is even.

Case 2: $n_{\max }$ is odd, and there are at least two nonoverlapping clusters in $G$ each of which is assigned only one distinct integer.

Case 3: $n_{\max }$ is odd, and there do not exist two nonoverlapping clusters in $G$ each of which is assigned only one distinct integer.

We consider the three cases one by one.

Case 1: $n_{\max }$ is even. In this case, clearly we can find $\frac{n_{\max }}{2}$ nonoverlapping clusters such that at least one of them is assigned only one integer. Among those $\frac{n_{\max }}{2}$ nonoverlapping clusters, let $x, y, z, a, b$, and $c$, respectively, denote the number of clusters that are assigned only integer " 1 ," only integer " 2 ," only integer " 3 ," both integers " 1 " and " 2 ," both integers " 2 " and " 3 ," and both integers " 1 " and " 3 ." Since the interleaving is an MCI, clearly $x+y+a \leq m-1$, $y+z+b \leq m-1, z+x+c \leq m-1$. So $2 x+2 y+2 z+a+b+c \leq 3 m-3$. Hence,

$$
x+y+z+a+b+c \leq 3 m-3-(x+y+z) \text {. }
$$

Since $x+y+z \geq 1$ and $n_{\max }=2(x+y+z+$ $a+b+c)$, we get

$$
\begin{aligned}
n_{\max } \leq 2[3 m-3- & (x+y+z)] \leq 6 m-8 \\
& =(N-1)[(m-1) N-1] .
\end{aligned}
$$

Case 2: $n_{\max }$ is odd, and there are at least two nonoverlapping clusters in $G$ each of which is assigned only one distinct integer. In this case, clearly we can find $\frac{n_{\max }-1}{2}$ nonoverlapping clusters among which there are at least two clusters each of which is assigned only one distinct integer. Among those $\frac{n_{\max }-1}{2}$ nonoverlapping clusters, let $x, y, z, a, b$, and $c$, respectively, denote the number of clusters that are assigned only integer " 1 ," only integer " 2 ," only integer " 3 ," both integers " 1 " and " 2 ," both integers " 2 " and " 3 ," and both integers " 1 " and " 3 ." Since the interleaving is an MCI, clearly, $x+y+a \leq m-1, y+z+b \leq m-1$, $z+x+c \leq m-1$. So

$$
2 x+2 y+2 z+a+b+c \leq 3 m-3 .
$$

Hence,

$$
x+y+z+a+b+c \leq 3 m-3-(x+y+z) .
$$

Since $x+y+z \geq 2$ and $n_{\max }=2(x+y+z+$ $a+b+c)+1$, we get

$$
\begin{aligned}
& n_{\max } \leq 2[3 m-3-(x+y+z)]+1 \leq 6 m-9 \\
&<(N-1)[(m-1) N-1] .
\end{aligned}
$$


Case 3: $n_{\max }$ is odd, and there do not exist two nonoverlapping clusters in $G$ each of which is assigned only one distinct integer. Let $x^{\prime}, y^{\prime}, z^{\prime}, a^{\prime}, b^{\prime}$, and $c^{\prime}$, respectively, denote the number of edges in $G$ whose two endpoints are both assigned integer " 1 ," are both assigned integer " 2 ," are both assigned integer " 3 ," are assigned integers " 1 " and " 2 ," are assigned integers " 2 " and " 3 ," are assigned integers " 1 " and "3." (Then $x^{\prime}+y^{\prime}+z^{\prime}+a^{\prime}+b^{\prime}+c^{\prime}=n_{\max }$.) It is simple to see that among $x^{\prime}, y^{\prime}$, and $z^{\prime}$, two of them equal 0 , and the other one is either 1 or 2 . So WLOG, we consider the following two subcases.

Subcase 3.1: $x^{\prime}=1$, and $y^{\prime}=z^{\prime}=0$. In this case, $a^{\prime} \leq 2 m-3$, because otherwise there will be $m$ nonoverlapping clusters in $G$ that are assigned only integers " 1 " and " 2 ." Similarly, $c^{\prime} \leq$ $2 m-3$. Also clearly, $b^{\prime} \leq 2 m-2$. If $a^{\prime}=2 m-3$ and $c^{\prime}=2 m-3$, then since there do not exist $m$ nonoverlapping clusters in $G$ that are assigned only one or two distinct integers, the MCI on $G$ can only take the form described as follows. In $G$, there are $a^{\prime}=2 m-3$ consecutive edges each of which has integers " 2 " and " 1 " assigned to its endpoints, which form a segment in the cycle $G$ that begins with a vertex assigned the integer " 2 ", and ends with a vertex assigned the integer "1." That segment is followed by an edge whose two endpoints both are assigned the integer " 1 ," then followed by $c^{\prime}=2 m-3$ consecutive edges each of which has the integers " 1 " and " 3 " assigned to its endpoints, and finally followed by $b^{\prime}$ consecutive edges each of which has the integers " 3 ", and " 2 " assigned to its endpoints, finishing the loop of edges in the cycle $G$. Then it is simple to see that $b^{\prime}$ cannot be even, which implies that $b^{\prime}<2 m-2$ here. So in any case, we have

$$
\begin{aligned}
a^{\prime}+b^{\prime}+c^{\prime} & <(2 m-3) \\
& +(2 m-2)+(2 m-3)=6 m-8
\end{aligned}
$$

So

$n_{\max }=x^{\prime}+y^{\prime}+z^{\prime}+a^{\prime}+b^{\prime}+c^{\prime}<6 m-7$.

Hence, $n_{\max } \leq 6 m-8=(N-1)[(m-1) N-1]$.

Subcase 3.2: $x^{\prime}=2$, and $y^{\prime}=z^{\prime}=0$. In this case, with arguments similar to those in Subcase 3.1 , we get $a^{\prime} \leq 2 m-4, c^{\prime} \leq 2 m-4$, and $b^{\prime} \leq 2 m-2$. So

$$
\begin{aligned}
n_{\max }= & x^{\prime}+y^{\prime}+z^{\prime}+a^{\prime} \\
& +b^{\prime}+c^{\prime} \leq 2+(2 m-4)+(2 m-2) \\
& +(2 m-4)=6 m-8 \\
= & (N-1)[(m-1) N-1] .
\end{aligned}
$$

So it has been proved that in any case

$$
n_{\max } \leq(N-1)[(m-1) N-1]
$$

In the following, we present an algorithm for generating MCIs on cycles. A distinct feature of this algorithm is that it needs to treat the cases " $n$ is even" and " $n$ is odd" somehow differently. Note that a Eulerian walk in a graph is a closed walk that passes every edge of the graph exactly once.

Algorithm 3: MCI on a cycle with constraints $L=2$ and $K=3$ Input: A cycle $G=(V, E)$ of $n$ vertices. Parameters $N, K, m$, and $L$, where $N \geq 3, K=3, m \geq 2$, and $L=2$.

Output: An MCI on $G$.

Algorithm:

1. If $n>(N-1)[(m-1) N-1]$, then there does not exist an MCI on $G$, so exit the algorithm.

2. If $n \leq N$, arbitrarily select $n$ integers in the set $\{1,2, \ldots, N\}$, and assign one distinct integer to each vertex, then exit the algorithm.

3. If $N<n \leq(N-1)[(m-1) N-1]$ and $n-(N-\overline{1})[(m-1) N-1]$ is even, then define a set $S$ as $S=\{(1,2),(2,3),(3,4), \ldots,(N-2, N-1),(N-1,1)\}$; if $N<n \leq(N-1)[(m-1) N-1]$ and $n-(N-\overline{1})[(m-1) N-1]$ is odd, then define a set $S$ as $S=\{(1,2),(2,3),(3,4), \ldots,(N-1, N),(N, 1)\}$.

Let $H=\left(V_{H}, E_{H}\right)$ be a graph with parallel edges that satisfies these four requirements: 1$)$ its vertex set is $V_{H}=$ $\left.\left\{u_{1}, u_{2}, \ldots, u_{N}\right\} ; 2\right)$ there is no loop in $H$, and all the edges in $H$ are undirected; 3 ) there are $n$ edges in $H$; 4) for any two vertices $u_{i}$ and $u_{j}$, if the unordered pair $(i, j)$ belongs to the set $S$, then the number of edges between them is odd and is no greater than $2 m-3$; otherwise, it is even and is no greater than $2 m-2$.

Find a Eulerian walk in $H, u_{k_{1}} \rightarrow u_{k_{2}} \rightarrow \cdots \rightarrow u_{k_{n}}$ (and finally back to $u_{k_{1}}$ ), that satisfies the following condition: for any two vertices, the walk passes all the edges between them consecutively.

For $i=1,2, \ldots, n$, assign the integer " $k_{i}$ " to the vertex $v_{i}$ in $G$, then exit the algorithm.

The following is an example of Algorithm 3.

Example 5: Assume $G=(V, E)$ is a cycle of $n=12$ vertices, and the parameters are $N=4, K=3, m=3$, and $L=2$. Therefore,

$$
N<n \leq(N-1)[(m-1) N-1]
$$

and

$$
n-\{(N-1)[(m-1) N-1]\}=-9
$$

is odd. Hence, Step 3 of Algorithm 3 is used to compute the interleaving, where the set $S$ is defined to be $S=\{(1,2),(2,3),(3,4),(4,1)\}$. Then we can choose the graph $H=\left(V_{H}, E_{H}\right)$ to be the one shown in Fig. 13(a). We can then (easily) find the following Eulerian walk in $H$ :

$u_{1} \rightarrow u_{3} \rightarrow u_{1} \rightarrow u_{2} \rightarrow u_{1} \rightarrow u_{2}$

$$
\rightarrow u_{4} \rightarrow u_{2} \rightarrow u_{4} \rightarrow u_{2} \rightarrow u_{3} \rightarrow u_{4}
$$

(then back to $u_{1}$ ). Corresponding to that walk, we get the MCI as shown in Fig. 13(b).

Theorem 6: Algorithm 3 correctly outputs an MCI on the cycle $G$.

The correctness of the preceding theorem should be clear once the proof of Theorem 2 is understood. Now we can present the necessary and sufficient condition for MCI to exist on cycles when $L=2$ and $K=3$. 
$\mathrm{n}=12, \quad \mathrm{~N}=4, \quad \mathrm{~K}=3, \quad \mathrm{~m}=3, \quad \mathrm{~L}=2$

(a)

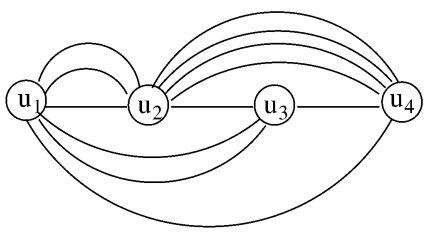

(b)

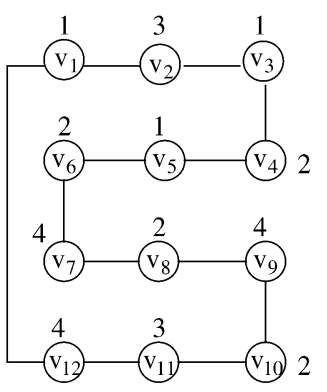

Fig. 13. (a) The graph $H=\left(V_{H}, E_{H}\right)$. (b) MCI on the cycle $G=(V, E)$.

Theorem 7: When $L=2$ and $K=3$, there exists an MCI on a cycle of $n$ vertices if and only if $n \leq(N-1)[(m-1) N-1]$.

\section{CONCLUSION}

In this paper, the MCI problem for paths and cycles is studied. Compared to traditional interleaving schemes, MCI has the distinct feature that the diversity of integers is required in multiple-instead of single-clusters. It has potential applications in data streaming, broadcast, and disk storage.

There exist many open problems in MCI. How to optimally construct MCI without the constraint that $K=L+1$ is still unknown. Also, in the MCI problem, the path/cycle can be replaced by more general graphs. Such extensions will help bring MCI into practice. We hope the techniques presented here will provide insights for further study.

\section{APPENDIX I \\ ON THE COMPLEXITY OF ALGORITHM 2}

When Algorithm 2 runs, it generates vertices in paths. We define this more rigorously in what follows. Algorithm 2 has three basic operations: 1) inserting vertices into an existing path to get a longer path; 2) combining two paths with overlapping vertices; 3) using Algorithm 1 to generate a path. For the first operation, we say that those vertices inserted into the existing path are newly generated vertices. For the second operation, we say that no vertex is newly generated. For the third operation, we say that all the vertices in the path output by Algorithm 1 are newly generated vertices. The complexity of Algorithm 2 is dominated by the total number of vertices generated while Algorithm 2 runs.

In this appendix, we shall prove that while Algorithm 2 runs, the total number of vertices generated is less than twice the order of the final output path $G=(V, E)$. The method is to prove the following sufficient condition: "while Algorithm 2 is running, if a vertex overlaps another vertex while the two paths they respectively belong to are combined, those two vertices will not overlap any more vertex later on." (Namely, for any vertex in the final output path $G=(V, E)$, it is the overlapping of at most two previously generated vertices.)

The recursive structure of Algorithm 2 is illustrated in Fig. 12. Let us consider an arbitrary one of the recursions, whose corresponding input parameters are $x, y+1, m, y$-namely, the output of this recursion is Algorithm2 $(x, y+1, m, y)$. (For the definition of Algorithm2 $(a, b, c, d)$, please see Fig. 12.) The output of this recursion is denoted by " $G$ " in the algorithm; and if $y \geq 3$, during this recursion, a set of paths denoted by " $B_{i}$ " and " $A_{i}$ " (for different values of $i$ ) will be created. Let us first prove the following lemma.

Lemma 7: If $y \geq 3$, then $B_{i}$ contains at least $2 y$ vertices, $A_{i}$ contains at least $2 y+2$ vertices, and $G$ contains at least $2 y+2$ vertices.

Proof of Lemma 7: We use induction. When $y=3, B_{i}$ is the output of another recursion-Algorithm2 $(i-1, y=$ $3, m, y-1=2)$. (Note that $m \geq 2$ and $i-1 \geq y=3$.) The path Algorithm2 $(i-1, y=3, m, y-1=2)$ is computed by calling Algorithm 1, so its order is

$(i-1-1)[(m-1)(i-1)-1]+2 \geq(i-2)^{2}+2 \geq 6=2 y$. $A_{i}$ is created by inserting at least $\left\lfloor\frac{2 y}{y-1}\right\rfloor=3$ vertices into $B_{i}$, so $A_{i}$ contains at least $2 y+3>2 y+2$ vertices. $G$ contains at least as many vertices as $A_{i}$. This serves as our base case.

Now assume the assertions of this lemma are true when $y \leq t-1$, and let us prove them for the case $y=t$. When $y=t$, $B_{i}$ is the output of another recursion-Algorithm2 $(i-1, y=$ $t, m, y-1=t-1)$; and by the induction assumption, Algorithm2( $i-1, y=t, m, y-1=t-1)$ contains at least $2(y-1)+2=2 y$ vertices. So $B_{i}$ contains at least $2 y$ vertices. $A_{i}$ is created by inserting at least $\left\lfloor\frac{2 y}{y-1}\right\rfloor \geq 2$ vertices into $B_{i}$, so $A_{i}$ contains at least $2 y+2$ vertices. $G$ contains at least as many vertices as $A_{i}$. That concludes this proof.

Now we can prove the "sufficient condition" mentioned in the second paragraph of this appendix. Assume in one of the recursions-whose corresponding input parameters are $x, y+1$, $m, y$-two paths $A_{i}$ and $A_{i+1}$ are combined; and let $u_{j}$ and $u_{j^{\prime}}$ be two vertices—respectively, in $A_{i}$ and $A_{i+1}$ — that overlap each other in that "combining" operation. In that recursion, for any integer $t, A_{t}$ contains at least $2 y+2$ vertices by Lemma 7; and when two paths are combined, only $y-1$ vertices are overlapped. So $u_{j}$ and $u_{j^{\prime}}$ do not overlap any other vertex in that recursion, and they are neither among the first $y+3$ vertices nor among the last $y+3$ vertices of the path output by this recursion (which is denoted by " $G$ " in the algorithm). Now assume this recursion is called as a procedure by a second recursion. The second recursion (whose input parameters are $*, y+2, m$, $y+1$ ) will insert vertices into the path output by the first recursion (with one new vertex inserted after every $y$ vertices of the path, scanning backward) to obtain a longer path-which we shall denote by $A^{\prime}$. So in the path $A^{\prime}$, there is at least one newly inserted vertex before $u_{j}$ and $u_{j^{\prime}}$ (which are now the same vertex) and at least one newly inserted vertex behind them. So $u_{j}$ and $u_{j^{\prime}}$ are neither among the first $y+4$ vertices nor among the last $y+4$ vertices of $A^{\prime}$. In the second recursion, the combining of two paths will overlap only $y$ vertices. So $u_{j}$ and $u_{j^{\prime}}$ will not overlap any other vertex in the second recursion. Similarly, $u_{j}$ 
and $u_{j^{\prime}}$ will not overlap any other vertex in future recursions. That concludes our proof.

\section{ACKNOWLEDGMENT}

The authors would like to thank the Associate Editor for his great diligence and the anonymous reviewers for their very helpful comments.

\section{REFERENCES}

[1] K. A. S. Abdel-Ghaffar, "Achieving the Reiger bound for burst errors using two-dimensional interleaving schemes," in Proc. IEEE Int. Symp. Information Theory, Ulm, Germany, 1997, p. 425.

[2] C. Almeida and R. Palazzo, "Two-dimensional interleaving using the set partition technique," in Proc. IEEE Int. Symp. Information Theory, Trondheim, Norway, 1994, p. 505.

[3] M. Blaum and J. Bruck, "Correcting two-dimensional clusters by interleaving of symbols," in Proc. IEEE Int. Symp. Information Theory, Trondheim, Norway, 1994, p. 504.

[4] M. Blaum, J. Bruck, and P. G. Farrell, "Two-dimensional interleaving schemes with repetitions," in Proc. IEEE Int. Symp. Information Theory, Ulm, Germany, 1997, p. 342.

[5] M. Blaum, J. Bruck, and A. Vardy, "Interleaving schemes for multidimensional cluster errors," IEEE Trans. Inf. Theory, vol. 44, no. 2, pp. 730-743, Mar. 1998.

[6] J. W. Byers, M. Luby, M. Mitzenmacher, and A. Rege, "A digital fountain approach to reliable distribution of bulk data," in Proc. ACM SIGCOMM'98, Vancouver, BC, Canada, Sep. 1998, pp. 56-67.
[7] T. Etzion and A. Vardy, "Two-dimensional interleaving schemes with repetitions: Constructions and bounds," IEEE Trans. Inf. Theory, vol. 48, no. 2, pp. 428-457, Feb. 2002.

[8] K. Foltz, L. Xu, and J. Bruck, "Scheduling for efficient data broadcast over two channels," in Proc. IEEE Int. Symp. Information Theory, Chicago, IL, Jun./Jul. 2004, p. 113.

[9] A. Jiang and J. Bruck, "Diversity coloring for information storage in networks," in Proc. IEEE Int. Symp. Information Theory, Lausanne, Switzerland, Jun./Jul. 2002, p. 381.

[10] A. Jiang, M. Cook, and J. Bruck, "Optimal $t$-interleaving on tori," in Proc. IEEE Int. Symp. Information Theory, Chicago, IL, Jun./Jul. 2004, p. 22.

[11] A. Mahanti, D. L. Eager, M. K. Vernon, and D. Sundaram-Stukel, "Scalable on-demand media streaming with packet loss recovery," in Proc. ACM SIGCOMM'01, San Diego, CA, Aug. 2001, pp. 97-108.

[12] Y. Merksamer and T. Etzion, "On the optimality of coloring with a lattice," in Proc. IEEE Int. Symp. Information Theory, Chicago, IL, Jun./Jul. 2004, p. 21.

[13] M. Naor and R. M. Roth, "Optimal file sharing in distributed networks," SIAM J. Comput., vol. 24, no. 1, pp. 158-183, 1995.

[14] D. A. Patterson, G. A. Gibson, and R. Katz, "A case for redundant arrays of inexpensive disks," in Proc. SIGMOD Int. Conf. Data Management, Chicago, IL, 1988, pp. 109-116.

[15] M. Schwartz and T. Etzion, Optimal 2-Dimensional 3-Dispersion Lattices (Lecture Notes in Computer Science). Berlin, Germany: Springer-Verlag, 2003, vol. 2643, pp. 216-225.

[16] A. Slivkins and J. Bruck, "Interleaving schemes on circulant graphs with two offsets," IEEE Trans. Inf. Theory, to be published.

[17] W. Xu and S. W. Golomb, "Optimal interleaving schemes for correcting 2-D cluster errors," in Proc. IEEE Int. Symp. Information Theory, Chicago, IL, Jun/Jul. 2004, p. 23. 